\title{
An Information Sampling Explanation for the In-Group Heterogeneity Effect
}

\author{
Elizaveta Konovalova \\ Max Planck Institute for Human Development \\ Berlin, Germany
}

\author{
Gaël Le Mens \\ Universitat Pompeu Fabra, Barcelona School of \\ Management and Barcelona GSE \\ Barcelona, Spain
}

\begin{abstract}
PSYCHOLOGICAL REVIEW
(C) American Psychological Association, 2019. This paper is not the copy of record and may not exactly replicate the authoritative document published in the APA journal. The final article is available, upon publication, at: http://doi.org/10.1037/rev0000160
\end{abstract}

\begin{abstract}
People often perceive their in-groups as more heterogeneous than their out-groups. We propose an information sampling explanation for this in-group heterogeneity effect. We note that people frequently obtain larger samples of information about in-groups than about out-groups. Using computer simulations, we show that this asymmetry in sample sizes implies the in-group heterogeneity effect under a wide range of assumptions about how experience affects perceived variability. This is the case even when perceived variability is the outcome of rational information processing, implying that the structure of the environment is sufficient to explain the emergence of the in-group heterogeneity effect. A key assumption of our explanation is that perceived group variability depends on the size of the sample observed about this group. We provide evidence in support for this assumption in two experiments. Our results considerably expand the scope and relevance of a prior sampling explanation proposed by Linville, Fischer, and Salovey (1989). They also complement other explanations that proposed that information about in-groups and out-groups is processed differently.
\end{abstract}

Keywords: information sampling, judgment bias, perception of variability, rational analysis.

\section{Introduction}

A large amount of research has shown that people frequently perceive their groups as more heterogeneous than groups to which they do not belong (Boldry, Gaertner, \& Quinn, 2007; Ostrom \& Sedikides, 1992; Rubin \& Badea, 2012). For example, Park and Judd (1990) found that students majoring in one subject judged students of other majors as less heterogeneous on characteristics such as extroversion or impulsiveness. Linville, Fischer, and Salovey (1989) found that Yale undergraduate students perceived college students as more heterogeneous in terms of friendliness than older adults. By contrast, members of a senior community in Florida perceived older adults as more heterogeneous in friendliness than college students. This "in-group heterogeneity effect" has been explained in several ways. One type of explanation invokes differences in how information about in-groups and out-groups is processed (Ostrom, Carpenter, Sedikides, \& Li, 1993; Ostrom \& Sedikides, 1992; Park \& Rothbart, 1982) or encoded (Judd \& Park, 1988; Linville et al., 1989; Linville \& Fischer, 1998; Park \& Judd, 1990).
A second type of explanation notes that people often have prior beliefs that the out-group is more homogeneous than the in-group (Park \& Hastie, 1987). A third type of explanation centers on the self as part of the in-group (Park \& Judd, 1990): Because the self is frequently seen as distinctive, this would contribute to a perception of in-group heterogeneity. A fourth type of explanation takes as a premise that heterogeneity is seen as a positive feature of social groups and that people want to have a more positive view of their in-groups than their out-groups. People would thus be motivated to see in-groups as more heterogeneous than out-groups (Ostrom \& Sedikides, 1992; Rubin \& Badea, 2012).

In this paper, we propose a new explanation for the ingroup heterogeneity effect. We note that people tend to obtain larger samples of observations about in-groups than about out-groups. For example, people are more likely to interact with others of the same ethnicity, gender, social class, or occupation (Marsden, 1987; J. M. McPherson \& SmithLovin, 1987; M. McPherson, Smith-Lovin, \& Brashears, 2006). We show that this asymmetry in sample sizes implies the emergence of the in-group heterogeneity effect as an inherent property of the probabilistic world to which people are 
exposed.

Key to our explanation is the fact that the variability of a sample of observations tends to increase with sample size. Consider for example the variance of a sample of $k$ independent draws from a standard normal distribution (with mean $\mu=0$ and variance $\left.\sigma^{2}=1\right)$. This sample variance is a random variable that can be written $\hat{\sigma}_{k}^{2}=Q /(k-1)$ where $Q$ is distributed according to a chi-squared distribution with $k-1$ degrees of freedom $\left(\chi_{k-1}^{2}\right)$. The mean of $Q$ is $k-1$. Two features of chi-squared distribution are noteworthy: $Q$ is rightskewed (the probability that the sample variance is lower than the mean is higher than 50\%) and the skewness is decreasing in $k$ (the skewness is equal to $\sqrt{8 /(k-1)}$ ). Overall this implies that the sample variance tends to underestimate the true variance $\left(\sigma^{2}=1\right): P\left(\hat{\sigma}_{k}^{2}<\sigma^{2}\right)>.5$. Crucially, the probability of underestimation decreases with sample size.

If people obtain larger samples about in-groups than about out-groups and the tendency to underestimate variability decreases with sample size, then the experienced variability of the in-group will tend to be larger than the experienced variability of the out-group. Under the reasonable assumption that perceived variability depends on experienced variability, this implies that people will tend to perceive in-groups as more variable than out-groups. More formally, consider an

(C)2019, American Psychological Association. This paper is not the copy of record and may not exactly replicate the final, authoritative version of the article. Please do not copy or cite without authors' permission. The final article will be available, upon publication in Psychological Review, via its DOI: 10.1037/rev000016. Elizaveta Konovalova, Center for Adaptive Rationality, Max Planck Institute for Human Development; Gaël Le Mens, Department of Economics and Business, Universitat Pompeu Fabra, Barcelona School of Management and Barcelona Graduate School of Economics. G. Le Mens benefited from financial support from Southern Denmark University and Grants PSI201341909-P, \#AEI/FEDER UE-PSI2016-75353, Ramon y Cajal Fellowship (RYC-2014-15035) from the Spanish MINECO, Grant IN[15]_EFG_ECO_2281 from the BBVA Foundation and ERC Consolidator \#772268 from the European Commission. E. Konovalova was funded by Spanish MINECO Grant PSI2013-41909-P to G. Le Mens. An early version of this project was part of E. Konovalova's doctoral dissertation. We are grateful for discussions with and comments by Jerker Denrell, Michael Hannan, Robin Hogarth, participants in the UPF Behavioral Science Workshop, the Behavioral and Management Research Breakfast at UPF, the 2017 TOM Meeting, the 2017 Organizational Ecology Conference, CogSci 2017 and 2018, EASP 2017, MathPsych 2017, SPUDM 2017, SJDM 2017. We also thank Deborah Ain for editing the manuscript. Some of the ideas presented in this paper served as a basis for short papers published in the Proceedings of Cogsci 2017 and 2018. Correspondence concerning this article should be addressed to E. Konovalova, Center for Adaptive Rationality, Max Planck Institute for Human Development, konovalova@mpib-berlin.mpg.de. agent and its perceived variability for the in-group, $V_{i n}$, and the out-group, $V_{\text {out }}$. We show that the agent is more likely to perceive the in-group as the more variable group:

$$
P\left(V_{\text {in }}>V_{\text {out }}\right)>P\left(V_{\text {in }}<V_{\text {out }}\right) \text {. }
$$

This explanation for the in-group heterogeneity effect operates at a level different from the explanations mentioned above. Whereas these focus on how the mind processes information, our explanation emphasizes the properties of the information samples that the mind processes-the input of mental operations (Brunswik, 1952; Fiedler \& Juslin, 2006; H. A. Simon, 1956).

Previous research has noted the importance of sample size in estimations of variability, both in general (Kareev, Arnon, \& Horwitz-Zeliger, 2002) and in the context of the in-group heterogeneity effect (Linville et al., 1989). But the theoretical arguments developed in these papers differ from ours. They focused on the properties of uncorrected sample variance as a statistical estimator of the variance of a distribution $\left(\sigma^{2}\right)$ :

$$
\hat{\sigma}_{u, k}^{2}=\frac{1}{k} \sum_{j=1}^{k}\left(x_{j}-\bar{x}_{k}\right)^{2},
$$

where $k$ is the sample size and $\bar{x}_{k}$ is the sample mean. They noted that this estimator is statistically negatively biased, especially when based on a small sample. To see this, consider the formula for the expected value of the uncorrected sample variance estimator:

$$
E\left[\hat{\sigma}_{u, k}^{2}\right]=\sigma^{2}-\frac{1}{k} \sigma^{2}<\sigma^{2} .
$$

If people's perception of group variability corresponds to the uncorrected sample variance, they will systematically underestimate the true variability. Moreover, the amplitude of the underestimation will diminish as sample size increases. If people obtain larger samples about the in-group than about the out-group, this implies

$$
E\left[\hat{\boldsymbol{\sigma}}_{u, \text { in }}^{2}\right]>E\left[\hat{\boldsymbol{\sigma}}_{u, \text { out }}^{2}\right],
$$

where $\hat{\sigma}_{u, \text { in }}^{2}$ and $\hat{\sigma}_{u, \text { out }}^{2}$ denote the uncorrected sample variances of the two groups. ${ }^{1}$ There is an in-group heterogeneity effect in that the perceived variability of the in-group is systematically larger than the perceived variability of the outgroup.

This explanation of the in-group heterogeneity effect is elegant and parsimonious. Yet its scope is limited by the fact that it assumes some form of biased information processing: People are assumed to rely on a statistically biased estimator of variance. This information processing bias, in turn,

\footnotetext{
${ }^{1}$ This argument also works for other biased estimators such as the probability of differentiation, also analyzed by Linville et al. (1989).
} 
implies a bias in estimated variability. Because the size of the bias in estimation depends on sample size, an in-group heterogeneity effect emerges. This explanation is thus not a purely environmental, or sample-based explanation. Rather, it invokes both a property of the learning environment (the asymmetry in sample sizes collected about in-group vs. outgroup) and limitations of information processing. To see this, note if we "de-bias" the sample variance, then the systematic difference in perceived variabilities disappears. To do so, we just add $1 / k$ to the sample variance, which produces the wellknown "corrected sample variance estimator":

$$
\hat{\sigma}_{c, k}^{2}=\frac{1}{k-1} \sum_{j=1}^{k}\left(x_{j}-\bar{x}_{k}\right)^{2},
$$

where $k$ is the sample size.

In contrast to the uncorrected sample variance, this estimator is statistically unbiased. For all sample sizes $k$ :

$$
E\left[\hat{\sigma}_{c, k}^{2}\right]=\sigma^{2}
$$

This implies that the expected sample variabilities of the two groups are the same:

$$
E\left[\hat{\sigma}_{c, i n}^{2}\right]=E\left[\hat{\sigma}_{c, o u t}^{2}\right],
$$

where $\hat{\sigma}_{c, \text { in }}^{2}$ and $\hat{\sigma}_{c, \text { out }}^{2}$ denote the sample variances of the two groups. $^{2}$

This line of argument seems to suggest that once we rely on an unbiased estimator of variance, the sampling argument breaks down. In this paper, we show that this is not the case if we characterize the in-group heterogeneity effect in terms of the probability that an individual believes the in-group to be the more variable group (eq. 1). With this characterization, a sampling asymmetry in favor of the in-group is sufficient to produce an in-group heterogeneity effect even if we do not assume biased information processing. In other words, an argument that focuses purely on the properties of the environment is sufficient to produce an in-group heterogeneity effect.

This is important because there exists scant evidence about the nature of the intuitive variability estimators people use when estimating group variability or making decisions that depend on perceived group variability. More precisely, there is no strong evidence that people rely on uncorrected sample variance (or any other particular measure of variability), although there have been some attempts to find out which estimator best corresponds to perceived variability in a nonsocial-cognition context (Kareev et al., 2002). ${ }^{3}$ From a theoretical perspective, demonstrating that an in-group heterogeneity effect emerges without assuming a particular form of biased information processing considerably extends the potential scope of sampling explanations for this important phenomenon.
In what follows, we first provide a rational analysis of the emergence of the in-group heterogeneity effect. This offers a justification, from first principles, for our focus on the corrected sample variance as opposed to other samplebased estimators of variability. Using computer simulations, we demonstrate that the in-group heterogeneity effect emerges. Because the rational analysis ensures the absence of information-processing biases, this provides a clear demonstration that the in-group heterogeneity effect can be an inherent consequence of the structure of the environment. We also show that a similar result holds when assuming that the agent is a Bayesian processor of information. In the section Other Measures of Group Variability, we report computer simulations that demonstrate that a similar pattern emerges for many other measures of group variability used in the prior literature on the in-group heterogeneity effect. In the section Sensitivity to Model Assumptions, we discuss how the nature of the predicted asymmetry in perceived group variabilities changes as we change the assumptions of our model, such as assumptions about memory or the structure of the environment. In particular, we demonstrate that under some conditions, our sampling approach can produce the opposite pattern: an in-group homogeneity effect. In the subsequent section, we examine existing evidence about how sample size affects perceived variability and report the results of two new experiments. Experiment 2, in particular, demonstrates that by manipulating the sizes of the samples collected about the in-group and the out-group, we can induce an in-group heterogeneity effect or the opposite effect. In the section on Implications for Social Cognition, we discuss the implications of our analyses for the use of stereotypes, perception of group complexity and the occurrence of extreme judgments about group members. Finally, in the section on Biases in the Perceived Variability of Probability Distributions, we discuss the implications of our approach for the perception of the variability of distributions.

\footnotetext{
${ }^{2}$ Although prior literature has noted that most estimators of variability are highly correlated (Pollard, 1984, cited in Kareev et al., 2002), the argument that focuses on the statistically biased nature of the estimator does not apply to unbiased estimators such as the corrected sample variance. Although corrected sample variance and uncorrected sample variance are highly correlated estimators, the argument only works for the uncorrected sample variance.

${ }^{3}$ Experiment 3 in Kareev et al. (2002) asked participants to predict the "difference in value between items to be drawn out of two populations" (p.291). The authors found that the best predictor of participants' judgments involved noncorrected sample variance. However, this finding provides only indirect support for a claim that perceived variability corresponds to uncorrected sample variance, because normative principles suggest that participants' responses should have been affected not only by the perceived variabilities of the two populations but also by other factors such as the perceived means.
} 


\section{A Rational Analysis}

To demonstrate that an argument that focuses purely on the structure of the environment can explain a systematic asymmetry in the perceived variabilities of the in-group and the out-group, we follow the principles of the rational analysis of cognition outlined by Anderson (1991). This approach assumes that behaviors and beliefs are solutions to an optimization problem. To formulate the optimization problem, the analyst should specify several elements: the goal of the cognitive system, the structure of the environment, what the cognitive system knows about the structure of the environment, ${ }^{4}$ and the computational constraints to which the cognitive system is subject.

We consider two model settings that differ in terms of the assumptions about the environment and what the agent knows about it. In the first setting, the true variabilities of the two groups are the same, unbeknownst to the agent. In the second setting, the true variabilities can differ but are picked from the same prior distribution (the second model is a Bayesian model). In both settings, the environment exogenously determines the samples of information accessed by the agent. ${ }^{5}$ The agent chooses a variability estimator that allows it to satisfy an accuracy goal. ${ }^{6}$

\section{Model 1}

Environment and Sampling Rule. Consider a setting where an agent forms beliefs about two social groups ( $g=$ in,out). The agent belongs to one of the two groups; this is the in-group. Both groups are characterized by a single feature dimension $X$. We denote by $f_{g}$ the distribution of feature $X$ in group $g$. In this baseline model, the feature dimension has the same distribution in the two groups: $f_{\text {in }}=f_{\text {out }}$. We assume this distribution to be normal, with mean 0 and variance 1: $\mu_{\text {in }}=\mu_{\text {out }}=0, \sigma_{\text {in }}^{2}=\sigma_{\text {out }}^{2}=1$. Importantly, this implies that the two groups have the same variability.

In each period, an agent samples one of the two groups. Each time it samples a group $g$, it obtains one observation $x_{t, g}$ of feature $X$. Successive observations of the focal feature for a group are independent realizations of the underlying random variable.

Let $r$ characterize the probability that an agent samples the in-group in period $t \geq 1$. We assume that $r$ is larger than 0.5: The agent is more likely to sample the in-group than the out-group. The sampling advantage for the in-group implies that the agent will gather larger samples of information about the in-group than about the out-group. ${ }^{7}$

The extent to which people sample the in-group more often than the out-group (the value of $r$ ) depends on aspects of the social environment such as racial or ethnic segregation or the degree of homophily in people's social networks. Currently available evidence suggests that in many environments, people interact predominantly with others of the same group (see Denrell, 2005, for a review). For example, in many cities in the United States and elsewhere, there is spatial segregation based on ethnicity (van Kempen \& Özüekren, 1998) or race (Massey \& Denton, 1989): People's immediate social environments largely consist of others of the same race or ethnicity. Analyses of social networks based on data collected in nationally representative panels of respondents indicate that this tendency is widespread (Marsden, 1987; M. McPherson et al., 2006; M. McPherson, Smith-Lovin, \& Cook, 2001). In terms of our model, this suggests that many environments correspond to $r>.5$.

To obtain more direct evidence supporting this assumption, we analyzed the racial composition of communities in the United States using the 2000 edition of the General Social Survey. These data were collected by the National Opinion Research Center at the University of Chicago and were based on a representative sample of U.S. citizens (Davis, Smith, \& Marsden, 2016) (GSS). In the survey, respondents were asked to report their race and indicate the percentage of different races and ethnicities in their communities. Because of the specific format of the racial identity question, we estimated the community structure only for white and black respondents. $^{8}$ We calculated the share of the reported percentage of the in-group members in the combined reported percentages of in-group and out-group members. For example, if a white person reported that their community was $50 \%$ white and $20 \%$ black, the resulting estimate is $\frac{50}{50+20}=0.71$. The average estimates across all black and white respondents are 0.82 and 0.54 respectively. The median values are a bit higher at 0.88 and 0.60 respectively. These estimates indicate that white people have more than four times more whites than blacks in their communities. The asymmetry is not as large for black people. This is not surprising because the group of white people is the majority group in the U.S. whereas the

\footnotetext{
${ }^{4}$ Although Anderson (1991) does not single out this element, it is crucial to the specification of our models and their characterizations as "rational."

${ }^{5} \mathrm{We}$ relax this assumption in subsequent sections.

${ }^{6}$ In both models, we assume that the accuracy goal includes the constraint that the estimator is unbiased. In doing so, we follow standard practice in statistics that assumes that 'good' estimators are unbiased. Yet, it is theoretically possible to relax this assumption, as is done in research on the bias-variance trade-off in supervised learning (e.g, Brighton \& Gigerenzer, 2012). This would likely produce different 'rational' variability estimators. We leave such analysis for future work.

${ }^{7}$ To ensure that variability estimates exist for both groups, we assume that the agent has sampled two observations from each group before the first period (to keep the formulas as simple as possible, we assume they are done in periods -1 and 0 ).

${ }^{8}$ When asked about their race, the respondents were given three choices: "White," "Black," and "Other." This formulation made the identification of the in-group other than 'White' and 'Black' impossible.
} 
group of black people is a minority group. We explore issues related to group sizes in the Sensitivity to Model Assumptions section.

In the analysis reported in this section, we use $r=0.75$. This means that the agent is three times more likely to sample from the in-group than from the out-group.

Knowledge of the environment. The agent knows that for each group the focal feature is distributed according to a normal distribution but did not know the means or variances of the distributions.

Goal. We assume that the agent has an epistemic motivation in the sense that it wants to have an accurate perception of group variability (Campbell, 1958; Yzerbyt, Judd, \& Corneille, 2004). In the context of our model, we assume that this means that the agent wants to have an estimator that is unbiased and minimizes mean square error. $V_{t, g}$ denotes the agent's variability estimator about group $g$ at time $t$. This estimator should be unbiased:

$$
E\left[V_{t, g}\right]=\sigma_{g}^{2}
$$

It should minimize the mean square error:

$$
\operatorname{MSE}\left(V_{t, g}\right)=E\left[\left(V_{t, g}-\sigma_{g}^{2}\right)^{2}\right] .
$$

In summary, we assume the agent is solving a constrained optimization problem: it is trying to find an estimator that minimizes the mean square error and is unbiased. In statistics, an estimator that is a solution to this constrained optimization problem is generally called the "best" estimator.9

Computational constraints. The agent has perfect memory and unlimited computing abilities. In the section Sensitivity to Model Assumptions, we analyze how relaxing these assumptions affects the predictions of the model.

\section{Model 2}

Model 2 is the same as Model 1 with a small difference in the specification of the environment: The two groups need not have the same variability.

Environment and Sampling Rule. The true variances for the two groups $\sigma_{\text {in }}^{2}$ and $\sigma_{\text {out }}^{2}$ are independent draws from a uniform distribution $U(0,1)$. With this assumption, the probability that the in-group is truly more variable than the outgroup is .5: $P\left(\sigma_{\text {in }}^{2}>\sigma_{\text {out }}^{2}\right)=P\left(\sigma_{\text {in }}^{2}<\sigma_{\text {out }}^{2}\right)=.5$. Thus, there is no prior for or against the in-group heterogeneity effect.

The sampling rule is the same as in Model 1.

Knowledge of the environment. The agent knows the distribution from which the true variances are picked. This is the (correct) prior distribution. The agent updates this prior based on its observations of the two groups using Bayes's theorem. For simplicity, we assume that the mean on the $X$ dimension is known and equal to $0 .^{10}$

Goal. The goal is the same as in Model 1.
Computational constraints. As in Model 1, we assume unlimited computational abilities.

\section{Solution to the Optimization Problem: The "Best" Esti- mators of Variance}

We now describe the solutions to these two versions of the optimization problem. These are well-known estimators of variance.

Model 1. The unique estimator that is the solution to the optimization problem is the corrected sample variance (Casella \& Berger, 2002, p. 346)

$$
V_{t, g}^{c}=\frac{1}{n_{t, g}-1} \sum_{j=-1}^{t}\left(x_{j, g}-\bar{x}_{t, g}\right)^{2} I_{j, g},
$$

where $I_{j, g}$ is an indicator variable equal to 1 if group $g$ is sampled in period $j$ and equal to 0 otherwise, $n_{t, g}$ is the number of samples $\left(n_{t, g}=2+\sum_{j=1}^{t} I_{j, g}\right), \bar{x}_{t, g}$ is the mean of the sampled observations at the end of period $t$, and $x_{j, g}$ is the observation in period $j$. The corrected sample variance is an unbiased estimator of variance that minimizes mean square error among unbiased estimator when the underlying distribution is a normal distribution. In that sense, it is the "best" estimator in this setting (Casella \& Berger, 2002).

Model 2. The estimator that is the solution to the optimization problem is the Bayesian estimator of variance. We denote by $V_{t, g}^{\text {Bayes }}$ the Bayesian estimator of the variance of group $g$ at the end of period $t$. This is the mean of the posterior distribution of the variance of the focal feature in group g.

\section{Summary}

The agent in our model makes a number of observations about one dimension $\mathrm{X}$ of the two groups. The crucial feature of the model is that the agent is subject to an environmental asymmetry in that it has access to a larger sample of observations about the in-group than about the out-group. There are no constraints on memory storage, retrieval or the agent's computational abilities. The agent has a clearly specified accuracy goal, and the variability (variance) estimate it uses is a solution to an optimization problem. We specified two versions of the optimization problem that differ in terms of the assumptions about the knowledge the agent possesses about the structure of the environment. These two versions of the optimization problem constitute the rational basis for the well-known corrected sample variance and Bayesian estimator of variance.

In the rest of the paper, we will discuss many variations on the design of Model 1. We will, therefore, refer to this model as the baseline model.

\footnotetext{
${ }^{9}$ It is sometimes more formally referred to as the UMVUE (Uniform Minimum Variance Unbiased Estimator).

${ }^{10}$ Similar results hold if the means are unknown.
} 


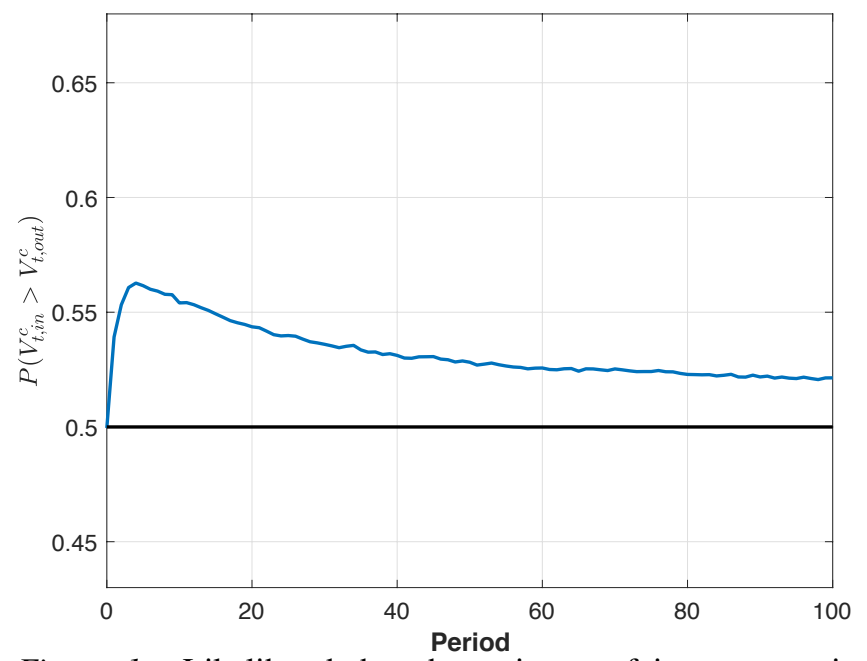

Figure 1. Likelihood that the estimate of in-group variability is higher than the estimate of out-group variability, $P\left(V_{t, \text { in }}^{c}>V_{t, \text { out }}^{c}\right)$, when the estimator of variability is the corrected sample variance (eq. 10). Based on $10^{5}$ simulations with $r=.75, \mu_{\text {in }}=\mu_{\text {out }}=0, \sigma_{\text {in }}^{2}=\sigma_{\text {out }}^{2}=1$.

\section{Results}

An in-group heterogeneity effect emerges with both estimators of variability.

Model 1: Corrected sample variance. We ran computer simulations of the model with $r=.75$. Figure 1 displays the likelihood that the estimate of the in-group variability is higher than the estimate of the out-group variability $P\left(V_{t, \text { in }}^{c}>V_{t, \text { out }}^{c}\right)$ as a function of the number of periods. It is higher than 0.5 for all periods after period 1 . In other words, the in-group tends to be perceived as more variable than the out-group even though the true variabilities are the same.

The likelihood first increases quickly and then decreases slowly with the number of periods. This asymmetry persists for a large number of periods; after 50 or even 100 periods the probability is still higher than 0.5 (it is 0.52 after 100 periods).

Model 2: Bayesian processor of information. We computed the mean of the posterior using the Markov chain Monte Carlo method explained in Shi, Griffiths, Feldman, and Sanborn (2010). Figure 2 displays the likelihood that the estimate of the in-group variability is higher than the estimate of the out-group variability $P\left(V_{t, \text { in }}^{\text {Bayes }}>V_{t, \text { out }}^{\text {Bayes }}\right)$ as a function of the number of periods. It is higher than 0.5 for all periods after period 1 despite the fact that the probability that the true variance of the in-group is larger than the true variance of the out-group is equal to 0.5 .

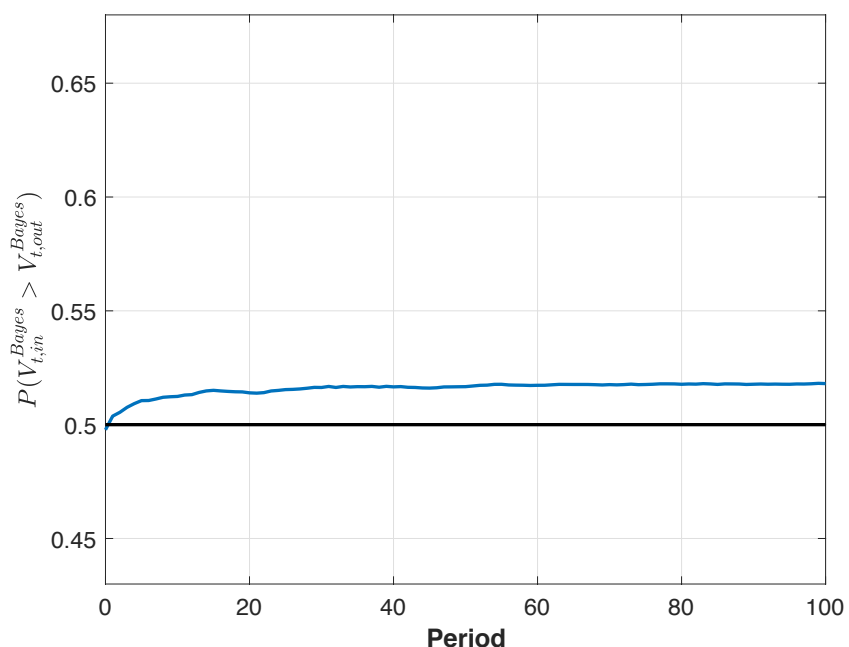

Figure 2. Likelihood that the estimate of in-group variability is higher than the estimate of out-group variability, $P\left(V_{t, \text { in }}^{\text {Bayes }}>V_{t, \text { out }}^{\text {Bays }}\right)$, when the measure of variability is the Bayesian posterior. Based on $10^{5}$ simulations with $r=.75$, $\mu_{\text {in }}=\mu_{\text {out }}=0, \sigma_{\text {in }}^{2} \sim U(0,1), \sigma_{\text {out }}^{2} \sim U(0,1)$.

\section{Intuition}

To develop an intuition for the emergence of the in-group heterogeneity effect in our simulations, we focus on Model 1 and examine what happens at a specific point in time. For concreteness, we focus on the end of period 15 and analyze the distributions of the corrected sample variances for the two groups. First, note that the in-group is sampled more times than the out-group (Figure 3, left panel). This is because of the assumed sampling advantage of the in-group $(r=.75)$. Second, note that the distributions of sampled variabilities for the two groups are right-skewed but to a different extent (Figure 3, middle panel). The distribution of the sample variance of the in-group $V_{15 \text {,in }}^{c}$ is less skewed than the distribution of the sample variance of the out-group $V_{15 \text {, out }}^{c}$. Overall, this implies that $V_{15 \text {,in }}^{c}$ tends to be larger than $V_{15, \text { out }}^{c}$, as shown by the distribution of $\Delta V_{t}^{c}=V_{t, \text { in }}^{c}-V_{t, \text { out }}^{c}$ (Figure 3, right panel): $P\left(V_{15, \text { in }}^{c}>V_{15, \text { out }}^{c}\right)=.55$. An in-group heterogeneity effect emerges: Most simulated agents experience the in-group as more variable than the out-group.

A similar intuition applies to Model 2, where the simulated agent was a Bayesian processor of information.

\section{Discussion}

Relation to explanations that focus on information processing. Most prior explanations of the in-group heterogeneity effect invoke differences in how information about in-group and out-group is processed. Here we discuss how our explanation differs from this prior work, using a taxonomy similar to that of Ostrom and Sedikides (1992). 

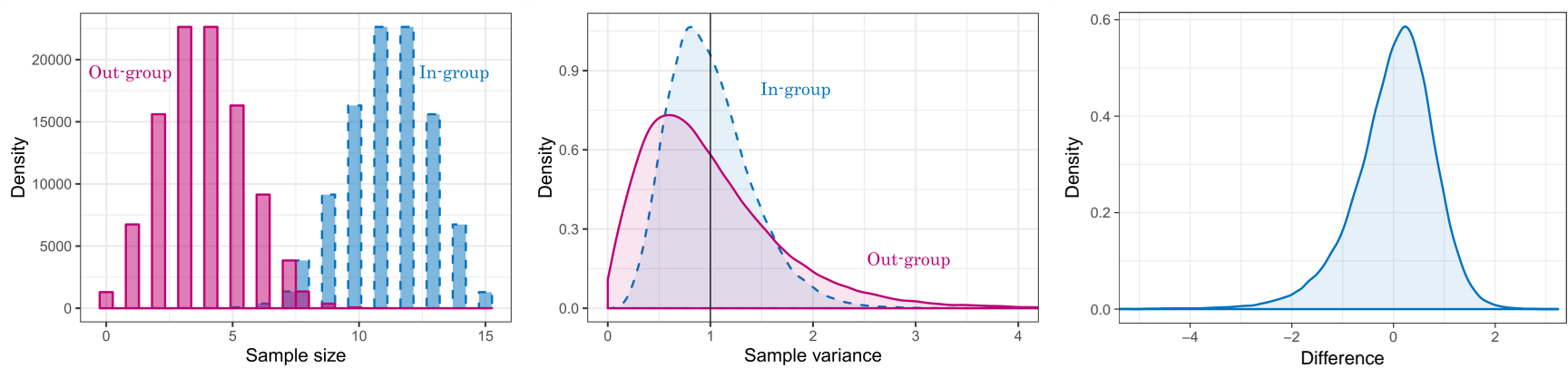

Figure 3. Model with corrected sample variance at the end of period 15. Left panel: Distribution of the sample sizes of the two groups. Middle panel: Distribution of variability estimates for the two groups, $V_{t, \text { in }}^{c}$ and $V_{t, \text { out }}^{c}$. The black vertical line denotes the true variance. Right panel: Distribution of difference in variability estimates $\Delta V_{t}^{c}=V_{t, \text { in }}^{c}-V_{t, o u t}^{c}$. Based on $10^{5}$ simulations with $r=.75, \mu_{\text {in }}=\mu_{\text {out }}=0, \sigma_{\text {in }}^{2}=\sigma_{\text {out }}^{2}=1$.

Several explanations for the in-group heterogeneity effect rely on motivated cognition (Kunda, 1990). The first invokes people's desires for positive identities: Those who want a positive social identity are motivated to view their in-groups more positively than other groups (Tajfel, 1982). Since heterogeneity is frequently perceived as a positive feature of social groups (Ostrom \& Sedikides, 1992), people are motivated to perceive the in-group as more heterogeneous than out-groups. A related explanation invokes people's desire for distinct identities: A more heterogeneous in-group allows people to see themselves as unique within the in-group, so people are motivated to see their in-group as heterogeneous (Pickett \& Brewer, 2001). Yet another explanation based on motivated cognition notes that it is easier to dehumanize more homogeneous groups (Brewer, 1999; N. Haslam, 2006). Therefore, if the out-group is perceived as less variable than the in-group, it is easier to justify negative attitudes and even cruel actions towards out-group members.

The second type of explanation notes that people tend to have prior beliefs that the out-group is more homogeneous. Park and Hastie (1987) showed that if participants first observed exemplars from a group followed by a description of its general characteristics, they perceived this group as more variable compared to when they observed that information in reversed order. This suggests that the prior about homogeneity affects how information is encoded. This finding implies an in-group heterogeneity effect under a (reasonable) assumption that people often learn descriptions of out-groups before interacting with some of their members (e.g., through stereotypes communicated by others in their environment) whereas they learn about in-groups through direct observations.

The third type of explanation hinges on the fact that the self is part of the in-group (Park \& Judd, 1990). The perception people tend to have of themselves as particularly differentiated and unique contributes to an impression that the in-group is more heterogeneous than the out-group.
The fourth type of explanations suggests that information about different groups is encoded and retrieved in different fashions. For example, Ostrom et al. (1993) found that information about in-group members is stored in categories related to individual information whereas information about out-group members is stored in categories related to stereotypical attributes. Therefore, when the information is recalled, the in-group tends to be associated with more individuating information compared to the stereotype-based homogeneous information about the out-group. Park and Judd (1990) went on to suggest that participants recall more extreme exemplars about in-groups than about out-groups; this indicates that memory search processes might differ across in-group and out-group.

These four types of explanation all emphasize features of information processing. By contrast, our explanation focuses on properties of the sample of information on which the mind operates. Because the two classes of explanation focus on different levels-our focus is on information sampling rather than information processing - they do not contradict each other. Rather, our explanation complements explanations that focus on information processing.

Characterization of the in-group heterogeneity effect. In contrast to the approach to the in-group heterogeneity effect typically adopted in the literature, we did not characterize the effect in terms of a difference in mean perceived variabilities (eq. 4). Rather, we focused on the likelihood that an agent perceives the in-group as the more variable (eq. 1). In other words, we focused on the skewness of the distribution of the difference in variability estimates, as illustrated by the right panel of Figure 3.

One reason for this characterization focusing on skewness was theoretical: We wanted to show that it was possible to characterize an emergent asymmetry in variability estimates even if information processing is unbiased. The sharpest way to characterize unbiased information processing is to assume (1) that the agent attempts to estimate the variance of the 
underlying distribution, and (2) that the variance estimator is statistically unbiased. Because the expected value of an unbiased estimator is the true value of the parameter, these two assumptions jointly imply that the expected variability estimate will be the true variance. And if the variances of the two groups are the same, there will be no difference in expected variability estimates. In other words, assuming that people rely on statistical unbiased estimators of variability logically implies that no in-group heterogeneity effect can be found if one defines it as a difference in means (eq. 4). Our model nevertheless leads to a systematic asymmetry in perceived group variabilities: In a large population of agents, most agents will tend to see their in-groups as more variable than their out-groups. Had we characterized the in-group heterogeneity effect in terms of a difference in means, we would have overlooked that fact that even unbiased information processing can lead to an asymmetry in perceived variabilities that can be interpreted as an in-group heterogeneity effect.

This asymmetry is a surprising consequence of the accuracy goal of the agents in our model. We assumed that agents wanted what statisticians call the "best" estimator of variability — an unbiased estimator that minimizes mean square errors. If we ignored this goal, it would be possible to construct a variability estimator that is not skewed and, consequently, does not produce an asymmetry in variability estimates like that of equation 4. Suppose, for example, that the agent takes the corrected sample variance and adds to it a random term that is skewed in a way that is exactly the opposite of the skew of the corrected sample variance. This variability estimator would be unbiased and not skewed. But it would not be the "best" estimator of variability because it would fail to minimize mean square error. Another strategy to avoid the asymmetry in variability estimates is to simply ignore sampled information. But this approach is unnecessarily extreme; the very fact that it ignores sampled information altogether makes it quite unreasonable. In summary, an asymmetry in perceived group variabilities is an inherent consequence of the conjunction of two factors: a larger sample of observation about the in-group than about the outgroup and a standard accuracy goal for the agent.

The second set of reasons for our characterization in terms of skewness pertains to empirical implications. Unlike our approach, a characterization in terms of a difference in means does not have clear implications for the propensity to perceive a group as more variable than another. In settings where perceived group variability affects choices (e.g., an agent decides whether to interact with members of group A or group B), only our approach leads to predictions about the alternative most likely to be chosen. This is particularly relevant to understanding settings where a set of people makes decisions through majority voting. This is because majority voting is insensitive to the strength of individual opinions.

For example, consider how members of a division within an organization perceive the diversity of their division as compared to that of the other divisions. Suppose that the organization is a university, the division is an academic department, and recruiting decisions are made based on majority voting. Suppose, moreover, that there exists a university policy to encourage diversity among academic staff members. The in-group heterogeneity effect, as per equation 1 , means that most members of the focal department will tend to over-estimate the relative diversity of their department as compared to that of the other departments. At the time of casting their votes, they will be unlikely to give much weight to the diversity criterion - after all, their department already seems more diverse than other departments.

Research on persuasion has found that people tend to be more convinced of a position when they have heard a larger number (Burnstein \& Vinokur, 1973, 1977) and a more diverse set of arguments supporting it (Chaiken, 1980, 1987; Soll, 1999). Take, for example, two groups whose leaders hold incompatible positions on a particular policy issue. Group members consult other people from their ingroups and out-groups to decide whether they should support their leaders on that issue. The in-group sampling advantage would imply that the agent collects more arguments supporting the position of the leader of their group. Furthermore, if equation 1 holds, most people will perceive these arguments as more diverse than the arguments supporting the position of the leader of the out-group. Therefore, most people will come to agree more with the opinion held by the leader of their group rather than with the opinion held by the leader of the out-group. In an anonymous vote for one of the two group leaders, people will thus be more likely to support the leader of their groups, thereby limiting the possibilities of leadership change in both groups and preventing the convergence of opinions across group boundaries. This prediction also holds even when the mechanisms typically invoked to explain more positive evaluations of in-group members, such as motivated cognition (e.g., Tajfel, 1982; Brown, 2000), or adaptive sampling (e.g., Denrell, 2005), do not operate.

In both examples, our sampling approach predicts that a majority of agents will see the in-group as the more diverse or variable (in terms of people's training or the arguments supporting the leader's opinion). Yet, the difference in perceived variabilities, for this majority of agents, tends to be smaller than the difference in perceived variabilities for the minority of agents who perceive the in-group as the less variable. Majority voting is generally not sensitive to opinion strength. This is why our model is most relevant to settings in which a population relies on majority voting to decide between options. But in settings where collective decision making is sensitive to the strength of opinions instead of just the proportion of people favoring one or the other opinion, our model does not make clear predictions. 
The rationality assumption. In this section, we assumed away potential limitations on memory and cognitive abilities, we assumed that people had accurate knowledge of the structure of the environment, and we assumed that the variability estimator they use is the solution to a clearly formulated optimization problem. We made all these assumptions in order to achieve theoretical clarity and parsimony. We aimed to demonstrate that in environments which lead agents to obtain larger samples about in-groups than about out-groups, the in-group heterogeneity effect is an inherent consequence of the structure of the environment.

We readily recognize that these assumptions are unrealistic. We do not claim that people are rational in the sense that to choose a variability estimator, they solve an optimization problem and derive from first principle that they should use the corrected sample variance or the Bayesian estimator of variance. Yet the fact that these unbiased estimators are those that allow agents to fulfill a realistic accuracy goal suggests that perceived variability is likely related to the corrected sample variance or the Bayesian estimator of variance.

At the same time, we believe it is unlikely that people's intuitive estimates of variability exactly correspond to either one of these rational estimators. One reason is that both of these estimators assume, unrealistically, that people have an infinitely large memory and perfect recall and give equal weight to all observations. Another reason that suggests people are unlikely to rely on the corrected sample variance is the large amount of evidence that people do not, and generally cannot, correct for the effects of sampling biases even when they are aware of them (for a review, see Fiedler, 2012).

To demonstrate the empirical relevance of our sampling approach, we thus need to show that the predicted in-group heterogeneity effect holds if we adopt more realistic assumptions about how people construct variability estimates based on their observations of a group. The evidence for this is scant, so we cannot simply evaluate whether the in-group heterogeneity effect predicted by our models would emerge with the (documented) group variability estimate people tend to use in naturally occurring environments. Instead, we focus on the measures of perceived group variability used in the existing empirical literature on the in-group heterogeneity effect. In every case, we construct a sample-based measure that closely corresponds to the measure discussed in the literature.

\section{Other Measures of Group Variability}

Empirical studies of the in-group heterogeneity effect have used many different measures of perceived group heterogeneity (see Boldry et al., 2007 for a review of the measures used in empirical research). Some studies elicited the perceived distribution of the focal trait in the groups, based on which the investigators constructed variability measures such as range (e.g., Quattrone \& Jones, 1980), variance (e.g.,
Linville et al., 1989), or the probability of differentiation (e.g., Linville et al., 1989). Other measures relied on the perceived similarity between group members (e.g., Alves, Koch, \& Unkelbach, 2016) or the number of subgroups that a participant can generate (e.g., Park, Ryan, \& Judd, 1992). Yet others relied on confusion in recall or recognition of the information about the groups (e.g., Ostrom et al., 1993). In this section, we consider the measures of subjective variability for which we could devise a characterization in terms of sample properties. For each one, we propose a corresponding sample-based measure and show that our sampling mechanism can lead to the emergence of an in-group heterogeneity effect.

The relevance of these analyses to the study of the ingroup heterogeneity effect presumes that perceived group variability depends on sample variability. Our (Konovalova \& Le Mens, 2018) recent review of existing empirical evidence supports this claim (Experiments $1 \& 2$ in Kareev et al., 2002, and Experiment 1 in Weber, Shafir, \& Blais, 2004). We also provided evidence based on a reanalysis of the Longitudinal Internet Studies for the Social Sciences (LISS) data. These data are from a representative sample of the Dutch population collected by CentERdata in collaboration with Galesic, Olsson, and Rieskamp (2012) for a project that explored the relationship between the social circles of the respondents (the individuals with whom they interact most frequently) and their perceptions of the national population as a whole. The survey asked respondents about 10 characteristics related to their financial situation, friendships, health, work stress, and education. The respondents reported their beliefs about the distribution of these characteristics in the population and in their social circle. For all these characteristics, we found a positive association between the variance of the perceived distribution of the national population and the variance of the distribution in the social circle (Konovalova \& Le Mens, 2018). These results indicate that perceived variability depends on sample variability under the assumption that the distribution of the social circle corresponds to the sampled distribution.

\section{Uncorrected Sample Variance}

The seminal paper by Linville et al. (1989) assumed that people relied on the uncorrected sample variance (see also Park \& Judd, 1990). The uncorrected sample variance for group $g$ at the end of period $t$ is:

$$
V_{t, g}^{u}=\frac{1}{n_{t, g}} \sum_{j=-1}^{t}\left(x_{j, g}-\bar{x}_{t, g}\right)^{2} I_{j, g}
$$

The components of this formula are the same as those in the formula for the corrected sample variance (eq. 10). Simulations of our model based on this estimator lead to a stronger in-group heterogeneity effect (with $r=.75$, Figure 4). For 


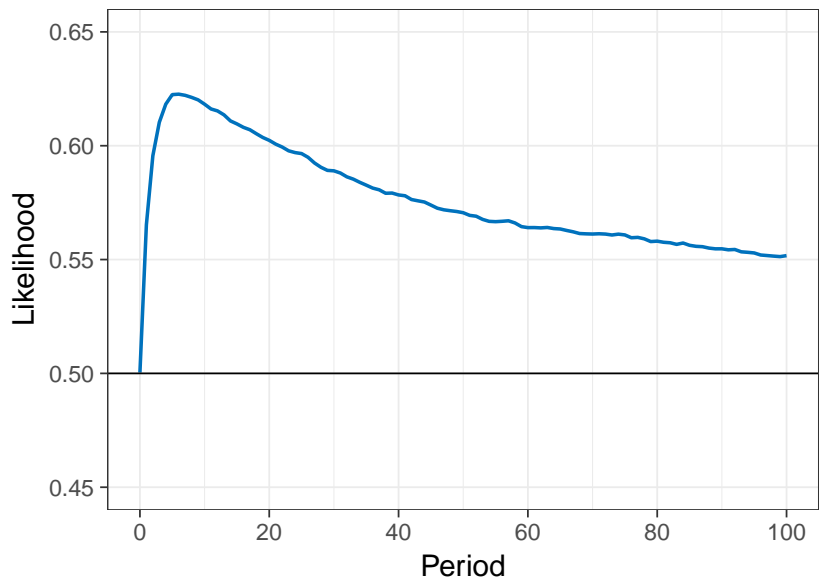

Figure 4. Likelihood that the estimate of in-group variability is higher than the estimate of out-group variability, $P\left(V_{t, \text { in }}^{u}>V_{t, \text { out }}^{u}\right)$, when the estimator of variability is the uncorrected sample variance (eq. 11). Based on $10^{5}$ simulations with $r=.75, \mu_{\text {in }}=\mu_{\text {out }}=0$, and $\sigma_{\text {in }}^{2}=\sigma_{\text {out }}^{2}=1$.

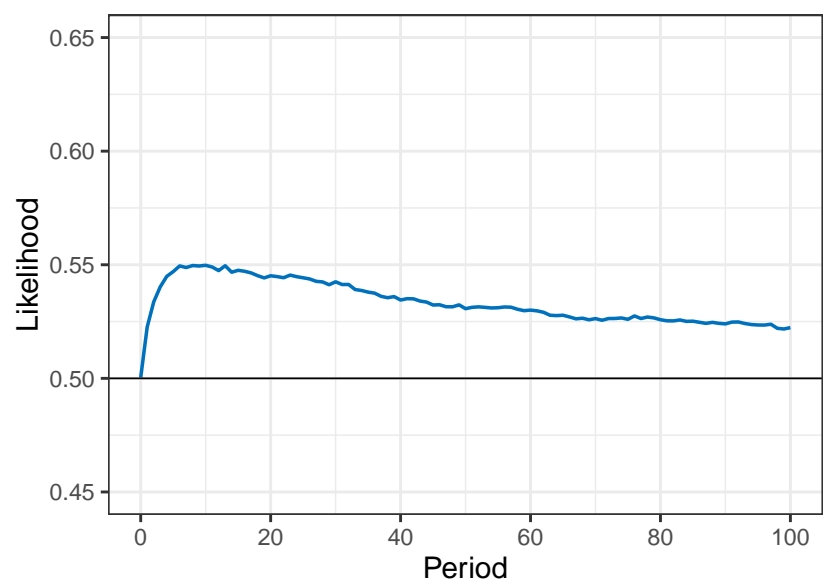

Figure 5. Likelihood that the estimate of in-group variability is higher than the estimate of out-group variability as a function of time for variance with differential weighting, $P\left(V_{t, \text { in }}^{e}>V_{t, \text { out }}^{e}\right)$. Each point is based on $10^{5}$ simulations with $r=.75, \mu_{\text {in }}=\mu_{\text {out }}=0, \sigma_{\text {in }}^{2}=\sigma_{\text {out }}^{2}=1$, and $\alpha=0.39$.

example, after 15 periods, the likelihood that the estimate of in-group variability is higher than the estimate of out-group variability is $P\left(V_{15, \text { in }}^{u}>V_{15, \text { out }}^{u}\right)=.61$. This number was .55 with the corrected sample variance.

\section{Sample Variance with Differential Weighting}

A study by Beach and Scopp (1968) proposed that when estimating the variance of a distribution, people are more sensitive to smaller deviations than to larger deviations from the mean. They proposed an alternative variance estimator where the deviations are taken not to the power of two but to another exponent:

$$
V_{t, g}^{e}=\frac{1}{n_{t, g}} \sum_{j=-1}^{t}\left|x_{j, g}-\bar{x}_{t, g}\right|^{\alpha} I_{j, g},
$$

where $\alpha>0$ is a parameter that can be estimated from data. The authors estimated it to be much smaller than 2: 0.39 . Such estimator gives more weight to smaller deviations and, therefore, will affect the magnitude of the variability asymmetry. We are not aware of any use of this measure in the literature on the perception of group variability, but since this measure of perceived variability (of a distribution) is one of the rare measures for which there is empirical support, it could correspond to how people form group variability judgments from their observations.

Figure 5 reports simulations of our model with this estimator of variability (with $r=.75$ ). An in-group heterogeneity effect emerges after the first period. For example, after 15 periods, $P\left(V_{15, \text { in }}^{e}>V_{15, \text { out }}^{e}\right)=.55$.

\section{Probability of Differentiation}

In several studies, participants were asked to recreate the distribution of a trait (e.g., friendliness) of the members of the groups over a set of "bins" (Judd \& Park, 1988; Judd, Ryan, \& Park, 1991; Linville et al., 1989). In these studies, a measure of variability called probability of differentiation was used. This is the probability that two randomly selected members of a group differ on the focal trait. In the original studies, the measure was based on discrete distributions with five values (from 1 to 5). The probability of differentiation was defined as

$$
P d=1-\sum_{i=1}^{5} p_{i}^{2}
$$

where $p_{i}$ is the density of value $i$ according to the elicited distribution $(i \in\{1,2,3,4,5\})$.

It is possible to adapt our model setup to this setting by using discrete (instead of continuous) feature distributions. As an illustration, we assume the focal feature has five levels, with probabilities $(0.1,0.2,0.4,0.2,0.1)$. This distribution corresponds to one of the settings analyzed in Linville et al. (1989).

Let $P d_{t, g}$ denote the probability of differentiation for the group $g$ based on the sampled distribution of the focal feature for this group until the end of period $t$. It is computed using eq. 13. There is an in-group heterogeneity effect when the in-group is more likely to be perceived as more variable (rather than less variable) than the out-group: $P\left(P d_{t, \text { in }}>P d_{t, \text { out }} \mid P d_{t, \text { in }} \neq P d_{t, \text { out }}\right)>$.5. $^{11}$

\footnotetext{
${ }^{11}$ There is generally a nonzero probability that the two groups have the same sample variability. Therefore, we need to use condi-
} 


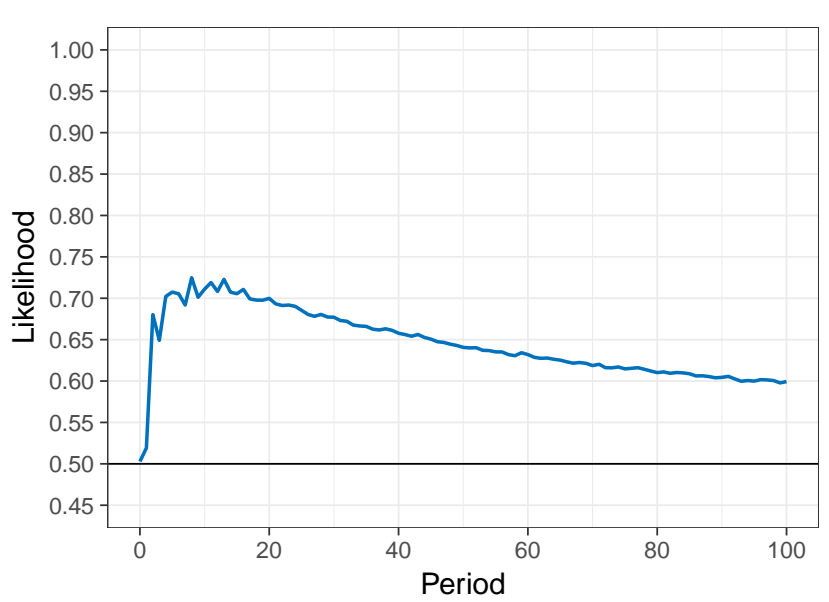

Figure 6. Likelihood that the estimate of in-group variability is higher than the estimate of out-group variability, $P\left(P d_{t, \text { in }}>P d_{t, \text { out }} \mid P d_{t, \text { in }} \neq P d_{t, \text { out }}\right)$ when the measure of variability is the probability of differentiation. Each point is based on $10^{5}$ simulations with $r=.75$ and a discrete distribution with five levels and the following frequencies: $(0.1,0.2,0.4,0.2,0.1)$.

Figure 6 reports simulations of our model with this estimator of variability (with $r=.75$ ). An in-group heterogeneity effect emerges after the first period. For example, after 15 periods, $P\left(P d_{15, \text { in }}>P d_{15, \text { out }} \mid P d_{15, \text { in }} \neq P d_{15, \text { out }}\right)=.7$. Additional simulations show that the strength of this effect increases with the sampling advantage for the in-group, $r$. For example, if $r=0.8$, then $P\left(P d_{15, \text { in }}>P d_{15, \text { out }} \mid P d_{15, \text { in }} \neq\right.$ $\left.P d_{15, \text { out }}\right)=.75$.

\section{Average Pairwise Similarity}

Another frequently used measure of group heterogeneity consists of asking participants to rate the similarity between group members. For example, Boldry and Gaertner (2006, p. 389) used the following question: "To what degree are all members of the group G similar in terms of feature Y?" (see also Badea, Brauer, \& Rubin, 2012; Quattrone \& Jones, 1980). One study used a spatial task where participants were asked to position group members on a screen; similarity was measured as the average distance between group members (Alves et al., 2016). This led to a measure that was the converse of similarity: the higher this average distance, the lower the average similarity.

We adapt this approach to our one-dimensional setting, using the absolute value of the difference between any two observations of the focal feature.

$$
D_{t, g}=\frac{1}{n_{t, g}\left(n_{t, g}-1\right)} \sum_{x_{1}, x_{2} \in O_{t, g}}\left|x_{1}-x_{2}\right|
$$

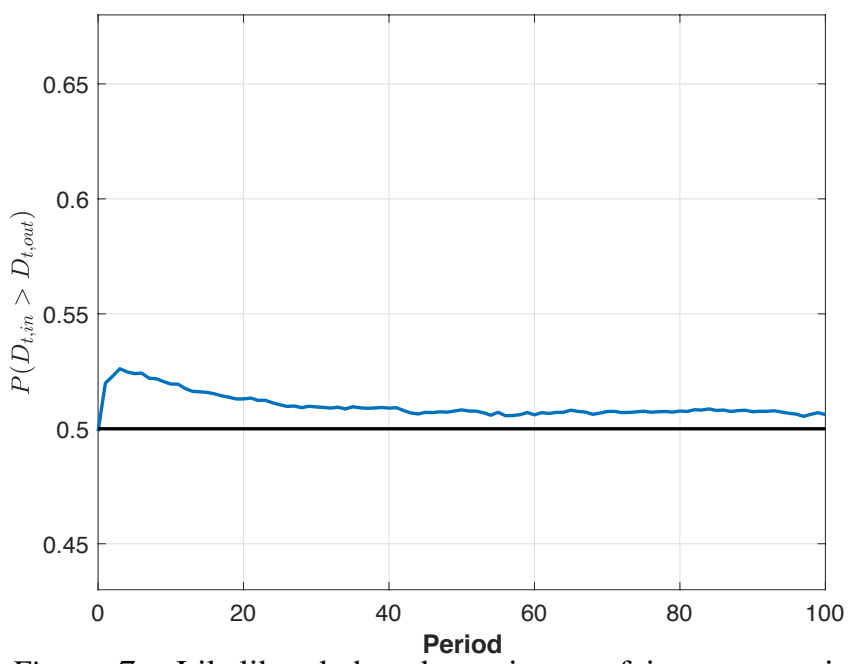

Figure 7. Likelihood that the estimate of in-group variability is higher than the estimate of out-group variability, $P\left(D_{t, \text { in }}>D_{t, \text { out }}\right)$, when the variability estimate is the average distance between pairs of group members (eq. 14). Based on $10^{5}$ simulations with $r=.75, \mu_{\text {in }}=\mu_{\text {out }}=0, \sigma_{\text {in }}^{2}=\sigma_{\text {out }}^{2}=1$.

Figure 7 reports simulations of our model with this estimator of variability (with $r=.75$ ). An in-group heterogeneity effect emerged after the first period: $P\left(D_{t, \text { in }}>D_{t, \text { out }}\right)>.5$.

\section{Range}

A widely used measure of variability is the range spanned by group members on the focal dimension (Boldry \& Gaertner, 2006; Jones, Wood, \& Quattrone, 1981; Quattrone \& Jones, 1980; Rubin, Hewstone, \& Voci, 2001). Usually, participants are asked to indicate how extreme a group member can lie on either side of the spectrum. Then, the low estimate is subtracted from the high estimate. In our model, we compute the experienced range as the maximum minus the minimum in the sample. Let $O_{t, g}$ denote the set of periods at which the agent sampled group $g$, until the end of period $t$ :

$$
R_{t, g}=\max _{j \in O_{t, g}} x_{j, g}-\min _{j \in O_{t, g}} x_{j, g} .
$$

Figure 8 reports simulations of our model with this estimator of variability (with $r=.75$ ). An in-group heterogeneity effect emerges after the first period: $P\left(R_{t, \text { in }}>R_{t, \text { out }}\right)>.5$ for $t \geq 1$. Moreover, this probability increased with the number

tional probabilities to characterize the asymmetry in perceived variability (conditioning on the variability estimators of the two groups being different from each other). As an illustration of the potential issue, note that it could happen that $P\left(P d_{t, \text { in }}>P d_{t, \text { out }}\right)<.5$ even though the in-group is likely to be seen as the more variable: $P\left(P d_{t, \text { in }}>P d_{t, \text { out }}\right)>P\left(P d_{t, \text { in }}<P d_{t, \text { out }}\right)$. In discrete settings, we thus say that there is an in-group heterogeneity effect when $P\left(P d_{t, \text { in }}>P d_{t, \text { out }} \mid P d_{t, \text { in }} \neq P d_{t, \text { out }}\right)>.5$ 


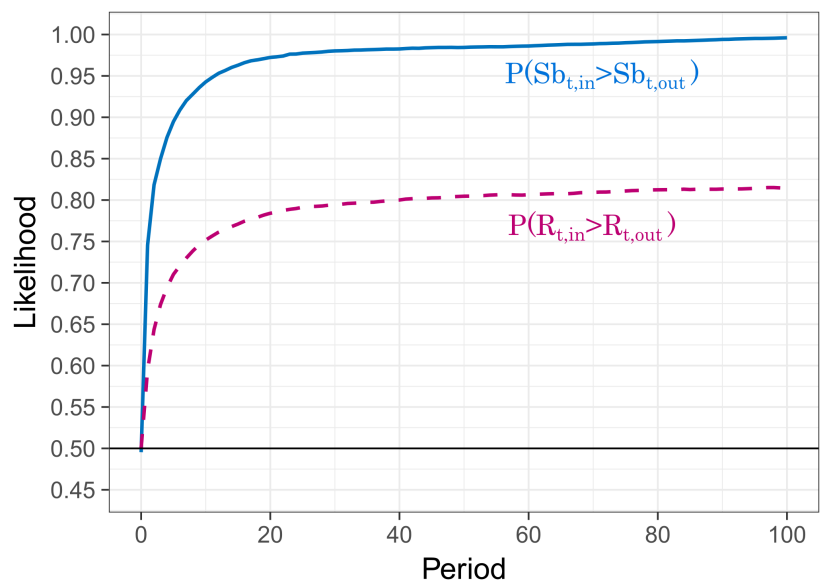

Figure 8. Likelihood that the estimate of in-group variability is higher than the estimate of out-group variability, when the variability estimate is the range $P\left(R_{t, \text { in }}>R_{t, \text { out }}\right)$ or the number of subgroups, $P\left(S b_{t, \text { in }}>S b_{t, \text { out }} \mid S b_{t, \text { in }} \neq S b_{t, \text { out }}\right)$. Based on $10^{5}$ simulations with $r=.75, \mu_{\text {in }}=\mu_{\text {out }}=0, \sigma_{\text {in }}^{2}=\sigma_{\text {out }}^{2}=$ 1 .

of periods. The intuition for this pattern is that the range cannot decrease when the sample size increases.

\section{Number of Subgroups}

In a few studies, participants were asked to generate "sorts or types" that could describe the groups (Linville, Fischer, \& Yoon, 1996; Park et al., 1992). In the studies that used this measure, an in-group heterogeneity effect emerged. Participants tended to generate more subgroups of the in-group than of the out-group.

A variation of our model adapted to this setting also produced this result. Research on categorization has shown that people tend to create additional categories when a new observation is far from observations in existing categories. The leading models are very sophisticated (e.g., Anderson, 1991; Sanborn, Griffiths, \& Navarro, 2010). Here we provide an illustration using a very simple model that captures the essence of this process. We assume that both the in-group and the out-group could be divided into up to 10 subgroups. We define boundaries between the subgroups using deciles of the normal distribution (we keep the assumption that the focal feature follows a standard normal distribution for the two groups). That is, an observation that is in the first decile would be in the first subgroup, an observation in the second decile would be in the second subgroup, etc.

We measure the number of types in a group $g$ by counting the number of subgroups that are being "hit" by the sample of observations for the group $g$. We denote the number of these subgroups by $S b_{t, g}$. For example, if all the observations fall in the same decile, group variability is minimal: $S b_{t, g}=1$.
If, by contrast, the observations span the 10 deciles, group variability is maximal: $S b_{t, g}=10$.

Figure 8 reports simulations of our model with this estimator of variability (with $r=.75$ ). An in-group heterogeneity effect emerges after the first period: $P\left(S b_{t, \text { in }}>S b_{t, \text { out }} \mid\right.$ $\left.S b_{t, \text { in }} \neq S b_{t, \text { out }}\right)$ for $t \geq 1$. The effect is very strong. For example, after 15 periods, $P\left(S b_{15, \text { in }}>S b_{15, \text { out }} \mid S b_{15, \text { in }} \neq\right.$ $\left.S b_{15, \text { out }}\right)=.96$ (with $r=.75$ ). Additional simulations show that the strength of the effect increases with the sampling advantage for the in-group, $r$. For example, if $r=0.8$, then $P\left(S b_{15, \text { in }}>S b_{15, \text { out }} \mid S b_{15, \text { in }} \neq S b_{15, \text { out }}\right)=.98$.

\section{Proportion of Group Members Who Possess a Trait}

In several studies, participants were asked to indicate the proportion of group members possessing a stereotypical trait (Boldry \& Gaertner, 2006; Park \& Judd, 1990; Park et al., 1992; Quattrone \& Jones, 1980; Ryan, Judd, \& Park, 1996). A higher percentage was interpreted as an indication of lower group heterogeneity.

It is possible to analyze this kind of setting with our model by assuming the focal feature is binary (absent or present) and that the probability that a group member possesses it is $p_{g}$. As a measure of perceived group variability one minus the proportion of observations with the focal feature:

$$
\operatorname{Pr}_{t, g}=1-\frac{1}{n_{t, g}} \sum_{j=-1}^{t} x_{j, g}
$$

where $x_{j, g}=1$ if the focal feature is present and $x_{j, g}=0$ if it is absent (as before, $n_{t, g}$ is the number of observations of group $g$ until the end of period $t$ ).

Suppose the feature is prevalent in the population, but equally stereotypical of both the in-group and the out-group: $p_{\text {in }}=p_{\text {out }}=.85$. Figure 9 reports simulations of our model with this estimator of variability (with $r=.75$ ). An ingroup heterogeneity effect emerges after the first period: $P\left(\operatorname{Pr}_{15, \text { in }}>\operatorname{Pr}_{15, \text { out }} \mid \operatorname{Pr}_{15, \text { in }} \neq \operatorname{Pr}_{15, \text { out }}\right)>.5$ for $t \geq 1$. After 15 periods, this is $P\left(\operatorname{Pr}_{15, \text { in }}>\operatorname{Pr}_{15, \text { out }} \mid \operatorname{Pr}_{15, \text { in }} \neq \operatorname{Pr}_{15, \text { out }}\right)=$ .54. Additional simulations show that the strength of this effect increases with the sampling advantage for the in-group, $r$. If the trait is rare, the opposite effect emerges: an in-group homogeneity effect. Suppose $p_{\text {in }}=p_{\text {out }}=.15$. After 15 periods, $P\left(\operatorname{Pr}_{15, \text { in }}>\operatorname{Pr}_{15, \text { out }} \mid \operatorname{Pr}_{15, \text { in }} \neq \operatorname{Pr}_{15, \text { out }}\right)=.46$ (with $r=.75)$. We return to the in-group homogeneity effect in the next section.

\section{Discussion}

So far, we have considered a number of measures of group variability used in the prior empirical and theoretical literature. For each measure, we proposed a way it could be constructed on the basis of the sampled observations of the group, and we showed that our sampling mechanism could 


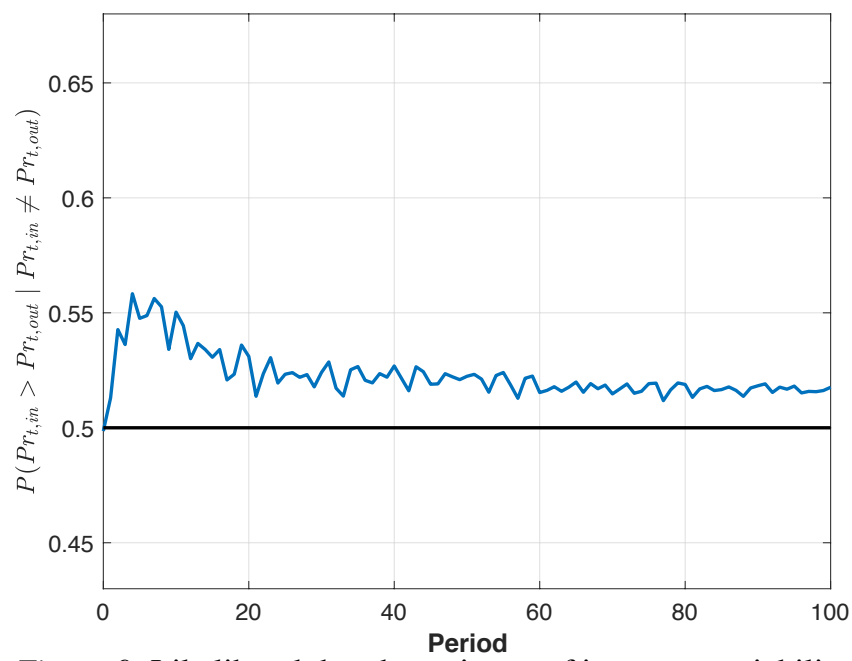

Figure 9. Likelihood that the estimate of in-group variability is higher than the estimate of out-group variability, $P\left(P r_{t, \text { in }}>\right.$ $\left.\operatorname{Pr}_{t, \text { out }} \mid P r_{t, \text { in }} \neq \operatorname{Pr}_{t, \text { out }}\right)$, when the measure of variability is the perceived proportion of group members that possess a trait (eq. 16). Based on $10^{5}$ simulations with $r=.75$, and $p_{\text {in }}=p_{\text {out }}=.85$.

produce an in-group heterogeneity effect for all these measures.

With some of the sample variability estimators, the model produces a substantially stronger effect than its baseline version with corrected sample variance (Model 1). This is the case with uncorrected sample variance, the probability of differentiation, range, and number of subgroups. Notably, all these estimators are biased with respect to the corresponding population level parameter (see our discussion of uncorrected sample variance above). This occurs because the estimators are subject to a negativity bias that becomes milder as sample size increases. For these estimators, the observed asymmetry in simulated group variability estimates is a result of the joint effect of the asymmetry in sample size and systematically biased information processing.

By contrast, the sample-based average pairwise similarity and the proportion of group members who possess a trait are unbiased estimators. Unsurprisingly, effect sizes are smaller in these cases. These analyses suggest that in settings where intuitive estimates of variability correspond to one of the biased estimators, analysts will observe a stronger in-group heterogeneity effect than in settings where they correspond to unbiased estimators. As mentioned in the introduction, our review of the literature on intuitive perception of variability identify little evidence about which variability estimator best corresponds to intuitive group variability. Additional research will need to address this issue.

When the variability estimator is biased, it is possible to compute the relative contributions of the sampling asymmetry and the bias in information processing to the overall asymmetry in perceived variabilities. Consider, for example, Figures 1 and 4, which display the perceived asymmetry in group variabilities in the case of the corrected sample variance (an unbiased estimator) and the uncorrected sample variance (a biased estimator). These two figures are based on the same model parameters. A comparison of these two figures indicates that close to $50 \%$ of the asymmetry in perceived variability can be attributed to the difference in sample size. The rest (the difference, on the y-axis, between the two graphs) can be attributed to biased information processing. The sampling asymmetry contributes to a substantial proportion of the total effect. ${ }^{12}$

In our analyses, we have assumed that agents pay attention to one focal feature of group members. This assumption was motivated by a desire to build a simple model and to relate to earlier research that focused on one-dimensional settings (Linville \& Fischer, 1998). Yet, when people form mental representations of group members and groups, they likely encode many features (Fiedler, 1996). It is possible to show that the impact of the sampling asymmetry in favor of the in-group can be larger in multidimensional settings.

Consider an extension of Model 1 to a multidimensional space. Suppose that $k$ features represent each group member. More precisely, each observation is a draw from a multivariate normal distribution. For simplicity, we assume that the features are uncorrelated with each other and that the variances on all dimensions are equal to $1 .{ }^{13}$ Instead of estimating variability based on the sample variance of the unique feature, the agent estimates the variances of each of the $k$ features. She does so based on the samples collected about the in-group and the sample collected about the outgroup. Suppose the agent perceives a group as more variable if he or she perceives a higher number of features as more variable for this group than for the other one. For example, consider a setting with 11 dimensions and suppose the agent perceives six features as more variable for the ingroup than for the out-group. Then the agent will judge the in-group as the more variable group. Ancillary simulations show that in such multidimensional setting the in-group heterogeneity effect is larger than in the one-dimensional case. After 15 periods, $P\left(V_{15, \text { in }}^{c}>V_{15, \text { out }}^{c}\right)=0.63$ (it was 0.55 in the one-dimensional setting). Moreover, the size of the effect increases with the number of dimensions: with 21 dimensions $P\left(V_{15, \text { in }}^{c}>V_{15, \text { out }}^{c}\right)=0.67$; and with 61 dimensions $P\left(V_{15, \text { in }}^{c}>V_{15, \text { out }}^{c}\right)=0.76$.

\footnotetext{
${ }^{12}$ This analysis does not imply that motivated cognition plays no role in explaining instances of the in-group heterogeneity effect. But because it is unclear what intuitive estimator people use when answering questions about group variability, it is not possible to identify the contribution of motivated cognition unless people observe samples of the same sizes about the two groups.

${ }^{13}$ The variance-covariance matrix is thus the identity matrix of dimension $k$.
} 
These results resonate with earlier work by Fiedler (1996) who showed that because the information about the in-group is denser than about the out-group, more unique features are extracted from a set of multidimensional vectors representing group members (see also Fiedler, Kemmelmeier, \& Freytag, 1999). There are many possible approaches to studying how sample size can affect perceived variability in multidimensional settings. Another possible approach would consider how people select features to form a mental representation of social groups in settings where the agent potentially perceives numerous dimensions of each group member. Prior research suggests that people will try to rely on a restricted set of dimensions (Niv et al., 2015). We conjecture that the mental representation of social groups will invoke a higher number of dimensions when the sample of observations about the group is larger. This, in turn, could explain the finding that people have mental representations about in-groups that consist of more feature dimensions than about out-groups (Linville, 1982). We leave formal analysis of such mechanisms for future research.

There exist a number of measures of group heterogeneity we have not discussed in this section because it is unclear how they can be characterized by sample properties. For example, in several studies, participants were asked to recall or recognize traits of group members (Lorenzi-Cioldi, 1998; Ostrom et al., 1993; Ratcliff, Hugenberg, Shriver, \& Bernstein, 2011; T. L. Stewart, Vassar, Sanchez, \& David, 2000). Higher confusion among group members was interpreted as lower perceived group heterogeneity. It is not clear how our sampling mechanism could explain the findings of these studies without invoking specific assumptions about the storage and retrieval of information in memory.

Next, we show how the strength of the in-group heterogeneity effect predicted by our model varies with changes in model assumptions other than the measure of variability.

\section{Sensitivity to Model Assumptions}

In the Rational Analysis section, we restricted our analyses to two sets of simulations with the same unique parameter value of $r=.75$ (the propensity to interact more frequently with the in-group). We also assumed perfect memory. In this section, we analyze how the size of the effect changes with model parameters and show that the predicted effect is robust to alternative assumptions about memory. Finally, we explore what happens when we change assumptions about how the environment affects the sampling of information about the in-group and the out-group.

An important finding is that our sampling model can also produce a pattern opposite to the in-group heterogeneity effect: an in-group homogeneity effect. The conditions under which this happens map well with empirical evidence that also produced this effect.

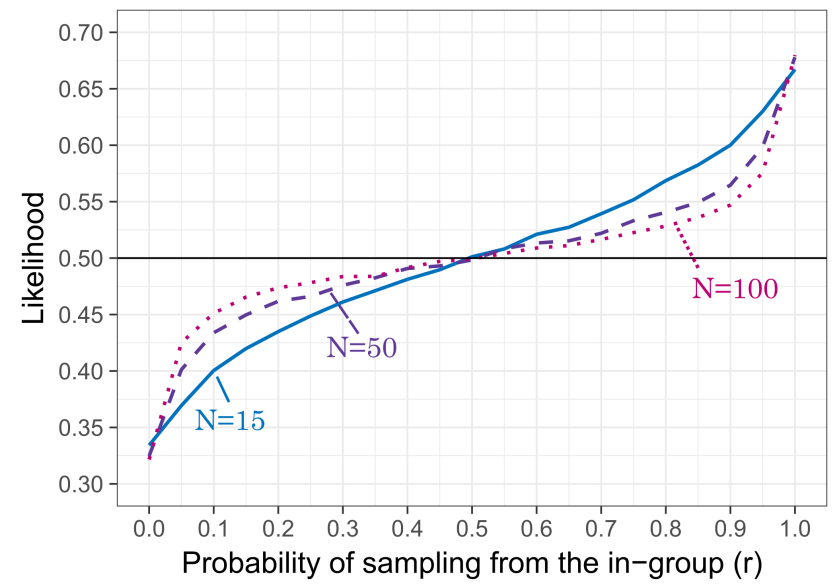

Figure 10. Likelihoods that the estimate of in-group variability is higher than the estimate of out-group variability, $P\left(V_{t, \text { in }}^{c}>V_{t, \text { out }}^{c}\right)$, after 15 (solid line), 50 (dashed line), and 100 periods (dotted line), as a function of the baseline probability of sampling the in-group $(r)$. The estimator of variability is the corrected sample variance (eq. 10). Based on $10^{5}$ simulations with $\mu_{\text {in }}=\mu_{\text {out }}=0 ; \sigma_{\text {in }}^{2}=\sigma_{\text {out }}^{2}=1$.

\section{Sensitivity to the Model Parameters}

Relative probabilities of sampling the in-group versus the out-group. A central assumption of our model is that the agent is more likely to interact with the in-group rather than the out-group. Figure 10 illustrates the likelihood $P\left(V_{t, \text { in }}^{c}>V_{t, \text { out }}^{c}\right)$ as a function of $r$. Unsurprisingly, a higher value of $r$ leads to a stronger effect-less so if the agent samples for longer.

By contrast, when $r<0.5$ (the out-group, not the ingroup, has a sampling advantage), the model predicts the opposite effect: An in-group homogeneity effect emerges $\left(P\left(V_{t, \text { in }}^{c}>V_{t, \text { out }}^{c}\right)<0.5\right)$. This fits well with existing evidence that an in-group homogeneity can emerge among members of a minority group (B. Simon \& Pettigrew, 1990a; Voci, Hewstone, Crisp, \& Rubin, 2008). The GSS survey data analyzed by Marsden (1987) suggests that members of a minority are frequently exposed to members of a majority (their out-group), especially when the minority is small. In the survey, Hispanic and black respondents listed more members of different racial groups among their closest contacts than did white respondents. At the extreme, members of a minority might interact more frequently with members of a majority group than with members of their own, minority, group. When this is the case, our model implies that the ingroup will tend to be judged as more homogeneous than the out-group.

Difference in true variabilities. To illustrate the implications of our sampling-based mechanism, we assumed 


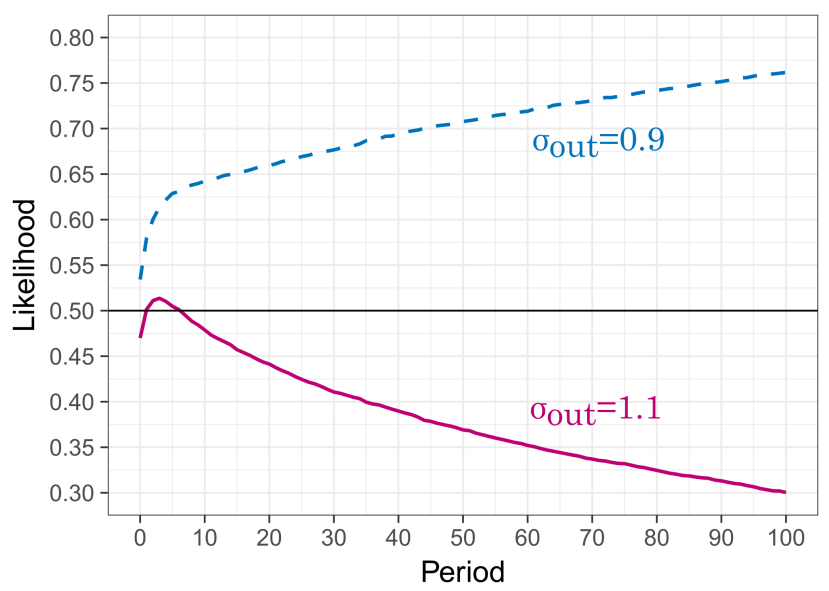

Figure 11. Likelihoods that the estimate of in-group variability is higher than the estimate of out-group variability, $P\left(V_{t, \text { in }}^{c}>V_{t, \text { out }}^{c}\right)$, for two levels of the true variability of the out-group: $\sigma_{\text {out }}^{2}=0.9^{2}, \sigma_{\text {out }}^{2}=1.1^{2}$. For both cases, the true variability of the in-group is $\sigma_{i n}^{2}=1$. The estimator of variability is the corrected sample variance (eq. 10). Based on $10^{5}$ simulations with $r=.75, \mu_{\text {in }}=\mu_{\text {out }}=0$.

that the true variabilities of the two groups were the same $\left(\sigma_{\text {in }}=\sigma_{\text {out }}\right)$. This does not have to be the case. For example, a focal feature that is stereotypical for one of the groups implies a difference in true variabilities. If the variabilities are different, our mechanism will imply the emergence of systematic errors in the perceived difference in variability. Suppose the variability of the in-group is higher than the variability of the out-group: $\sigma_{\text {in }}^{2}>\sigma_{\text {out }}^{2}$. In this case, most agents will perceive the difference in variabilities as higher than it really is: $P\left(V_{t, \text { in }}^{c}-V_{t, \text { out }}^{c}>\sigma_{\text {in }}^{2}-\sigma_{\text {out }}^{2}\right)>.5$. Moreover, the proportion of agents who perceive the in-group as the more variable group will be higher than when the true variabilities are the same. As an illustration, suppose $\sigma_{\text {in }}=1>\sigma_{\text {out }}=0.9$ (Figure 11). After 15 periods, $P\left(V_{15, \text { in }}^{c}-V_{15, \text { out }}^{c}>\sigma_{\text {in }}^{2}-\sigma_{\text {out }}^{2}\right)=$ .53. The probability of perceiving the in-group as more variable is $P\left(V_{15, \text { in }}^{c}>V_{15, \text { out }}^{c}\right)=.65$. It was .55 when the true variabilities were the same.

If the feature is stereotypical for the in-group, however, the difference in variability will result in an in-group homogeneity effect. This prediction is consistent with existing findings. In Experiment 1 by S. A. Haslam, Oakes, Turner, and McGarty (1995), Australians indicated from a list the traits that "seem the most typical of people" from Australia and the United States. Participants were asked to choose five of these traits and to provide the percentages of people who possess that characteristic in each country. The five traits chosen for Americans were regarded as stereotypical of Americans and counter-stereotypical of Australians. Similarly, the traits chosen as stereotypical of Australians were considered counter-stereotypical of Americans. Note that these definitions are specific to each participant, as every participant independently indicated which traits they regarded as stereotypical of each nation. Consider a trait listed as typical of Australians. Participants indicated their estimated percentage of Australians and Americans with this trait. The stated percentages of Australians were higher than the stated percentages of Americans. In other words, Australians perceived their in-groups as more homogeneous than their outgroups on stereotypical traits (assuming that group homogeneity is defined as the proportion of group members who possess a given trait).

To see how our approach relates to this kind of setting, consider the version of our model that includes the proportion of group members who possess a trait (analyzed in Section Other Measures of Group Variability). Suppose that the feature is binary and that $85 \%$ of the in-group members and $50 \%$ of the out-group members possess it $\left(p_{\text {in }}=.85 ; p_{\text {out }}=\right.$ $.5)$. The in-group is less variable than the out-group on this trait, and so an in-group homogeneity effect will emerge even if there is a sampling advantage for the in-group. For example, with $r=.75$ there is an in-group homogeneity effect in all periods. The in-group is less likely to be perceived as the more variable: $P\left(P r_{t, \text { in }}>P r_{t, \text { out }} \mid P r_{t, \text { in }} \neq P r_{t, \text { out }}\right)<.5$. After 15 periods, this probability is .08. A similar pattern occurs if there is no sampling asymmetry $(r=.5)$ because of the difference in true variabilities.

To capture this kind of situation with our model based on normally distributed features, it is enough to assume that the true variability of the in-group is lower than the true variability of the out-group: $\sigma_{\text {in }}^{2}<\sigma_{\text {out }}^{2}$. In this case, an in-group homogeneity effect could emerge if the difference in true variabilities is large enough: $P\left(V_{t, \text { in }}^{c}>V_{t, \text { out }}^{c}\right)<.5$. Suppose $\sigma_{\text {in }}=1<\sigma_{\text {out }}=1.1$. After 15 periods, $P\left(V_{15, \text { in }}^{c}>V_{15, \text { out }}^{c}\right)=$ .46 (see Figure 11).

\section{Assumptions about Memory}

In the sections above, we assumed away constraints on memory, and we assumed that the perceived variability of a group depended on the whole set of observations sampled from the environment about that group. The realism of these assumptions is questionable. There exists a large amount of evidence that when people learn from a sequence of observations they are subject to recency biases (see Denrell, 2005, for a review). Other papers have suggested that when people are asked for variability estimates, they might recall a limited sample of relevant observations from their memories and construct the variability estimate based on this small sample (e.g., Juslin, Winman, \& Hansson, 2007; Kareev et al., 2002).

Recency-weighted sample variance. We analyze a model where the agent stores an estimator of variability and updates it sequentially based on additional sampled observations. This approach is similar to models of belief updating 


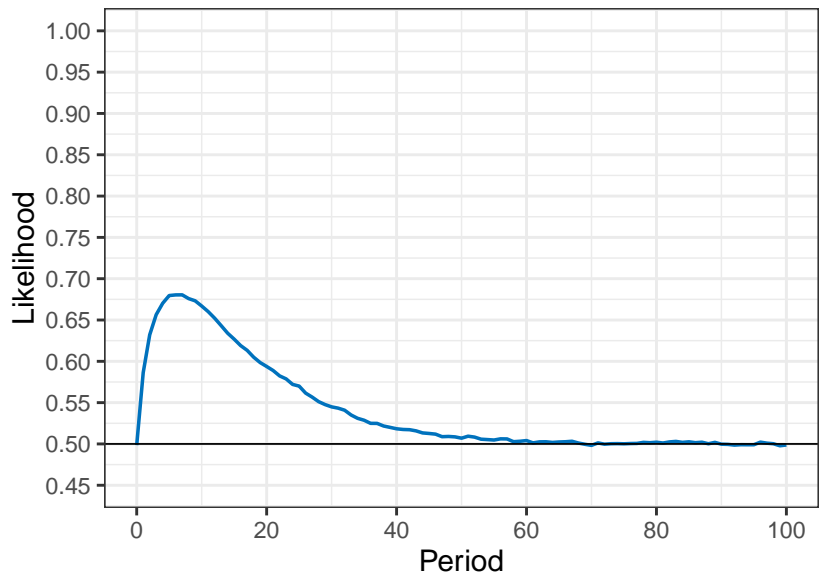

Figure 12. Likelihood that the estimate of in-group variability is higher than the estimate of out-group variability, $P\left(V_{t, \text { in }}^{r}>V_{t, \text { out }}^{r}\right)$, when the estimator of variability is the recency-weighted sample variance (eq. 17). Each point is based on $10^{5}$ simulations with $r=.75, \mu_{\text {in }}=\mu_{\text {out }}=0, \sigma_{\text {in }}^{2}=$ $\sigma_{\text {out }}^{2}=1$ and $b_{x}=0.3$.

that are commonly used in investigations of attitude formation (e.g. Denrell, 2005; Hogarth \& Einhorn, 1992; March, 1996).

To remain as close as possible to our baseline model, we assume that the agent aims to estimate the variance of the observed dimension for each group. Let $V_{t, g}^{r}$ be the estimate of the variance at the end of the period $t$ and $\hat{x}_{t, g}$ be the estimate of the mean. If the agent samples group $g$ in period $t$, the agent updates its estimates as follows:

$$
V_{t, g}^{r}=\left(1-b_{x}\right)\left[V_{t-1, g}^{r}+b_{x}\left(x_{t, g}-\hat{x}_{t-1, g}\right)^{2}\right],
$$

and:

$$
\hat{x}_{t, g}=b_{x} \hat{x}_{t-1, g}+\left(1-b_{x}\right) x_{t, g},
$$

where $b_{x} \in[0,1]$ is the weight of the most recent observation. If the group is not sampled, the estimators of mean and variance do not change. Note that this model implies an exponential memory decay. The strength of the decay increases with the size of the parameter $b_{x}$.

Figure 12 reports simulations of our model with this estimator of variability (with $r=.75$ ). An in-group heterogeneity effect emerges after the first period: $P\left(V_{t, g}^{r}>V_{t, g}^{r}\right)>.5$. The effect is less persistent than in the baseline simulations, however. This is because the assumed recency weighting implies limited memory capacity. And when the agent has sampled both groups enough to reach the limit, the sampling asymmetry does not imply a corresponding asymmetry in variability estimates.

Sampling from long-term memory. Research on the production of confidence intervals suggests that these are likely constructed from samples of observations retrieved from long-term memory. In an important paper, Juslin et al. (2007) proposed a model of how this might happen: the naïve sampling model. We see subjective confidence intervals as specific kinds of variability assessment; we, therefore, apply the naïve sampling model to a setting in which hypothetical study participants would judge which of two groups is the more variable. The naïve sampling model makes three basic assumptions:

1. "Each judgment elicits retrieval of a small sample of similar observations from long-term memory that become active in short-term memory.

2. The sample size is constrained by short-term memory capacity.

3. People directly use sample properties to estimate population properties." (Juslin et al., 2007, pp. 681-682)

We adopt these three assumptions. As in our baseline model, we assume that the focal agent observed one dimension of each group and constructs a variability estimate of each group on this dimension.

The true distribution of the feature in a group corresponds to the "objective environmental distribution" (OED) in the naïve sampling model. Juslin et al. (2007) specify the memory sampling process as follows:

In long-term memory, a subset of the target values in the OED is stored, and these observations define the subjective environmental distribution (SED). The target values of a sample of $n$ observations in the SED are retrieved to produce a subjective sample distribution (SSD). In the spirit of the naive intuitive statistician, we assume that the SSD is a random sample from the SED. (pp. 683-684)

In these terms, the sampling asymmetry discussed so far in the paper is an asymmetry that concerns the SED, the samples collected from the environment. According to the naïve sampling model, the variability estimates are based on the SSD, a sample of observations in short-term memory. Suppose the size of this sample is at most $n_{w}$ observations, which reflects the short-term memory capacity. We consider a setting where a person is asked which of two groups is the more variable. We assume that each observation stored in longterm memory is equally likely to be retrieved into short-term memory (independently of the group to which it belongs).

As an illustration, suppose that $n_{w}=7$. We make this assumption because it corresponds to the typical number of chunks of familiar information that can be stored in working memory (Miller, 1956). We assume $r=.75$, as in simulations of the baseline model. After 15 periods the amplitude 
of the in-group heterogeneity effect is close to what was obtained with the baseline model: $P\left(V_{15, \text { in }}^{c}>V_{15, \text { out }}^{c}\right)=.56$ (this quantity was .55 with the baseline model). The predictions of the two models differ when the number of periods is large, however. Suppose that there are 100 periods. In the limited memory model, the size of the effect would be the same as with 15 periods: $P\left(V_{100, \text { in }}^{c}>V_{100, \text { out }}^{c}\right)=.56$. But in the baseline model, the size of the effect is smaller than with 15 periods: $P\left(V_{100, \text { in }}^{c}>V_{100, \text { out }}^{c}\right)=.52$.

The fact that the effect is larger with the limited memory model results from two sampling asymmetries: an environmental sampling asymmetry and a resulting memory sampling asymmetry. The environmental sampling asymmetry is the asymmetry that operates in the baseline model and its variations discussed in the previous two sections. It affects the relation between OEDs and SEDs. The memory sampling asymmetry results from a conjunction of two factors. The first factor is the fact that in long-term memory there are more in-group observations than out-group observations. This is produced by the environmental sampling asymmetry. The second factor is the assumption that observations stored in long-term memory are retrieved independently of the group to which they correspond and with equal probability.

After a large number of periods, the two SEDs are based on large samples and thus the environmental sampling asymmetry in favor of the in-group is no longer very consequential for the asymmetry in the variabilities of the SEDs, as illustrated in our analysis of the baseline model (Figure 1). Yet, the memory sampling asymmetry in favor of the in-group remains consequential for the asymmetry in the variabilities of the SSDs, because the SSDs are based on small samples of systematically different sizes.

Existing research on overconfidence has shown that different types of questions trigger different memory sampling processes (Juslin et al., 2007). Similarly, we conjecture that the format of the questions used to elicit the in-group heterogeneity effect likely affects the size of the effect. We speculate that questions that ask an individual to compare the variabilities of two groups will trigger the memory sampling mechanisms posited above ( $n_{w}$ observations, of which some will be from the in-group and some from the out-group).

A different memory sampling mechanism would likely operate if study participants were asked the perceived variability of the two groups separately. In this case, up to $n_{w}$ observations from the in-group are retrieved from long-term to short-term memory when assessing the variability of the ingroup. And up to $n_{w}$ observations from the out-group are retrieved from long-term to short-term memory when assessing the variability of the in-group. Ancillary simulations show that if the same number of observations about each group are retrieved, there is no in-group heterogeneity effect implied by our sampling mechanism (with both corrected and uncor- rected sample variance). This does not imply that we would expect not to observe any in-group heterogeneity effect in this kind of setting, but rather that an observed asymmetry would likely be the outcome of differential processing of information about the in-group and the out-group.

Several studies have suggested that memory processes could explain the in-group heterogeneity effect (Ostrom et al., 1993; Park \& Judd, 1990). They proposed that there are systematic differences in how the information about ingroups and out-groups is retrieved. However, our analysis suggests that even if there is no difference in retrieval processes, a limited working memory capacity could contribute to explaining the in-group heterogeneity effect for some question formats.

\section{Sensitivity to Assumptions about the Structure of the En- vironment}

Adaptive sampling of groups. In the baseline model, we assumed that the sampling probabilities were exogenously set for the agent: The agent's experience did not affect its relative propensities for sampling one group or the other. Yet, in many settings, the valence of past experiences with a group affects an individual's propensity to sample the group again. When people have had a positive experience with a group, they are more likely to interact again with members of this group. Conversely, a negative experience makes them less likely to interact again with members of this group. A large amount of research has provided evidence for this pattern of approach and avoidance, called "adaptive sampling" in recent literature (for a review, see Denrell, 2005).

We analyzed a version of our model consistent with this observation. In this setting, whenever the agent samples a group, it updates its attitude toward the group based on the valence of its experience. It also makes an observation of the focal feature $X$ (with the same properties as in our baseline model). Let $A_{t, \text { in }}$ be the attitude toward the in-group and $A_{t, \text { out }}$ the attitude toward the out-group. The probability that the agent samples the in-group in period $t$ depends on its attitude as follows:

$$
P_{t, \text { in }}=l+(1-l) \frac{e^{s A_{t, \text { in }}}}{e^{s A_{t, \text { in }}}+e^{s A_{t, o u t}}},
$$

where $s$ characterizes the sensitivity of the sampling likelihood to the current attitude and $l \in[0,1]$ is a parameter that corresponds to the sampling advantage of the in-group. The higher $l$ is, the higher the sampling advantage for the ingroup. When $l$ is close to 1 , the agent frequently samples the in-group even if it has a negative attitude toward it $\left(A_{t, \text { in }}\right.$ is low). When $l$ is close to 0 , information sampling is mostly driven by the attitudes towards the two groups.

Simulations reported in Appendix A show that an ingroup heterogeneity effect also emerged in this setting. Ancillary analyses showed that the effect tended to be stronger 
when sampling was more sensitive to the valence of past experiences $(s)$. This is because in this case, the asymmetry in the sizes of the samples collected about the two groups was amplified.

Similarity-based sampling of people. Existing research on social networks provides evidence that people tend to create ties with similar others-this phenomenon is known as the "homophily principle" (Galesic et al., 2012; Marsden, 1987; J. M. McPherson \& Smith-Lovin, 1987). We can analyze the implications of this phenomenon for the emergence of the in-group heterogeneity effect by analyzing a version of our model in which the sampling behavior of the focal agent does not directly depend on group membership but rather on feature-based similarity.

Suppose there is a large population with two groups of equal size. From the perspective of a person in this population, half of the population belongs to the in-group and half belongs to the out-group. Each member of the society has two features, $X$ and $Y ; X$ can be observed without sampling whereas sampling is necessary to discover $Y^{14}$. For example, $X$ could pertain to physical appearance, whereas $Y$ could pertain to personality. This distinction is similar to the distinction between "proximal" and "distal" objects in construal level theory (Liberman, Trope, \& Wakslak, 2007; Trope \& Liberman, 2010). 15 Here we build on Fiedler's (2007) observation that agents obtain information about distal and proximal objects differently.

We assume that $Y$ and $X$ are both distributed according to normal distributions. The distribution of $Y$ is the same for members of the in-group and members of the out-group. The distributions of $X$, differ across groups, however. For in-group members, $X$ follows a $N(1,1)$ distribution. For outgroup members it follows a $N(0,1)$ distribution. Consider an agent, $i$. The assumption that people are more likely to interact with similar others than with dissimilar others is implemented by assuming that the probability that agent $i$ samples an agent $j$ in period $t$ decreases with the distance between the two agents on dimension $X$ :

$$
P_{t, j}=\frac{e^{-s\left|x_{i}-x_{j}\right|}}{\sum_{k} e^{-s\left|x_{i}-x_{k}\right|}},
$$

where $s$ characterizes the sensitivity of the sampling probability to similarity.

Simulations reported in Appendix A show that this model leads to the emergence of an in-group heterogeneity effect: The sample variability for the in-group tends to be higher than the sample variability for the out-group. As in other versions of our model, this results from the fact that the agent tends to obtain larger samples of observations about the ingroup. In this case, this sampling asymmetry is driven by the combination of two factors: the fact that the groups differ on the proximal dimension ( $X$ follows different distributions for in-group and out-group) and the fact that people tend to sam- ple others similar to them on this proximal dimension. The effect becomes stronger when the difference between the $X$ distributions is larger.

Groups of different sizes. In the baseline model, we implicitly assumed that groups were infinitely large. In reality, however, groups have finite sizes that generally differ from each other. Differences in group sizes have implications for the strength of the in-group heterogeneity effect. Suppose that the in-group is the larger of the two groups (it is the majority group). The difference in group sizes has two parallel effects that reinforce each other: first, the agent is more likely to sample members of the (larger) in-group; second, the ingroup, due to its larger size, is also more likely to be more variable than the out-group. The latter effect was absent in the simulations of the simple model (we assumed that the two groups had the same variability); therefore, in this setting the effect will be stronger than in our baseline case. Simulations confirmed this intuition. Suppose $r=.75, N_{\text {in }}=50, N_{\text {out }}=$ 10. After 15 periods, $P\left(V_{15, \text { in }}^{c}>V_{15, \text { out }}^{c}\right)=.62$. This value was .55 with the version of Model 1 analyzed at the beginning of the paper. (See Appendix A for details).

Now suppose that the in-group is the smaller one (the minority group). This difference in size implies it is likely less variable than the out-group. The nature of the asymmetry in perceived group variabilities will depend on the sampling advantage of the in-group. If the in-group has a strong sampling advantage, an in-group heterogeneity effect will emerge. But if the sampling advantage of the in-group is not as strong, an in-group homogeneity effect will emerge. To illustrate this, let us assume $N_{\text {in }}=10$ and $N_{\text {out }}=50$. For most values of $r$, an in-group homogeneity effect will emerge. For example, with $r=.75$ after 15 periods $P\left(V_{15, \text { in }}^{c}>V_{15, \text { out }}^{c}\right)=.47<.5$. However, for very high values of $r$, the sampling advantage compensates for the asymmetry in group sizes. For example, with $r=.9, P\left(V_{15, \text { in }}^{c}>V_{15, \text { out }}^{c}\right)=.5$.

These results predict that an in-group homogeneity effect will tend to emerge in minorities. But if the minority is strongly isolated from the majority ( $r$ is very high), an ingroup heterogeneity effect will prevail.

Distribution of the focal feature. The nature of the asymmetry in perceived group variabilities depends on the nature of the distribution of the focal feature. In most analyses reported so far, we assumed it was normally distributed. Some naturally occurring environments are better described by skewed or even bimodal distributions (Galesic et al., 2012; Pachur, Hertwig, \& Rieskamp, 2013). Ancil-

\footnotetext{
${ }^{14}$ Here we define sampling specifically as an interaction with a group member (e.g., a conversation). Therefore, we do not treat observing perceptual information $X$ by the agent as sampling.

${ }^{15}$ Construal level theory defines "proximal" and "distal" objects by their distance to the observer in their psychological space. While "proximal" objects are in the closer vicinity, "distal" ones are removed.
} 
lary simulations suggested that the in-group heterogeneity effect emerges with most unimodal distributions (although we could not formally prove that unimodality is a sufficient condition for the effect to emerge). When the distribution is not unimodal, the opposite effect can emerge: an in-group homogeneity effect. The intuition for this result is that when the distribution is bimodal, sample variance does not increase with sample size, but rather tends to decrease.

Suppose the distribution of the focal feature is a $\operatorname{Beta}(0.2,0.2)$, for both groups. After 15 periods, $P\left(V_{15, \text { in }}^{c}>\right.$ $\left.V_{15, \text { out }}^{c}\right)=.47$ (with $r=.75$ ). It is noteworthy that this quantity is lower than .5. The support of the $\operatorname{Beta}(0.2,0.2)$ distribution is $[0,1]$. It has a peak at 0 and a peak at 1 . When the sample of observations is small, it is likely that all observations will be close to one of the two peaks. But as the sample size increases, it is more likely that some intermediary values (close to the midpoint, 0.5) will be sampled. The sample variance thus decreases with sample size.

We are not aware of any existing study of the in-group heterogeneity effect that focused on features with bimodal distributions. Testing this distinctive prediction of our model is an interesting avenue for future research.

\section{Discussion}

We analyzed the sensitivity of the predictions of our sampling-based mechanisms to three classes of factors: model parameters, assumptions about memory, and assumptions about the structure of the environment. We identified a number of factors that affect the strength of the effect. Perhaps more importantly, we found that our mechanism can also explain an effect opposite to the in-group heterogeneity effect: an in-group homogeneity effect. We found that it will occur for features that are stereotypical of the in-group and when the in-group is a minority-predictions consistent with existing empirical evidence.

Yet our sampling approach cannot provide an alternative explanation for all existing findings about the in-group heterogeneity effect. An important assumption of our model is that the structure of the environment implies a sampling advantage for the in-group (probability to sample from the in-group $r>0.5)$. In other words, an asymmetry in sample sizes is necessary for the emergence of the difference in perceived group variabilities. Our sampling approach is thus unable to account for instances of the in-group heterogeneity effect in settings where there is no asymmetry of sample sizes. For example, an in-group heterogeneity effect has been found in experiments using a minimal group paradigm, in which participants are randomly assigned to a group during the experiment and asked to report the variability of a target group (either their group or the other one). In one such study, Rubin and Badea (2007) gave participants a dot estimation task where participants were asked to estimate the number of dots on a slide. After this task, the participants were as- signed to a group of "underestimators" or "overestimators" and asked to complete the range task for a number of traits for the target group. There was no asymmetry in sample sizes between the two groups. This implies that the difference in perceived group variability can only be produced by differences in how the information about the two groups is processed. Understanding how our sampling-based mechanism and information-processing mechanisms interact in creating the in-group heterogeneity effect is an interesting avenue for future research.

\section{Sample Size, Perceived Variability and In-Group Heterogeneity Effect: Empirical Evidence}

Our sampling explanation for the in-group heterogeneity effect relies on the observation that perceived variability tends to increase with sample size. In this section, we review existing evidence for this association and provide new evidence in two experiments.

\section{Existing Evidence}

Linville et al. (1989) asked Yale undergraduate students to estimate the distribution of their classmates in an introductory psychology class on five characteristics: likability, average number of hours per day spent studying outside of class, SAT scores, typical mood, and friendliness (Experiment 4). Each characteristic had seven levels. For each characteristic, participants were asked to indicate the percentage of their classmates that fall into each of the seven levels. The authors elicited the perceived distributions three times: near the beginning, at the midpoint, and near the end of the semester. They found that perceived variability increased with experience: The linear trend was positive and significant for both variance of the perceived distribution and the probability of differentiation.

Experiment 2 in Kareev et al. (2002) provides a more direct test of the hypothesis that perceived variability tends to increase with sample size. Participants saw two populations of equal variance (this was unknown to the participants) and were asked to indicate which of the two was the less variable. Unbeknownst to the participants, the two populations had the same distribution. They saw a sample from each population. For one population, participants saw the whole population (28 items); this was the "large sample population." For the other, they drew a random sample of seven items; this was the "small sample population." The majority of participants selected the small sample population as the less variable one. Participants also completed an incentivized task where the optimal choice was to select the less variable population (they were told that two items would be drawn from the selected population and they would receive a bonus if they were close enough). Again, the majority of participants selected the small sample population. Overall, these results 
indicate that the participants perceived the small sample population as less variable than the large sample population.

These two studies provide evidence that the perceived variability of a distribution increases with sample sizes. The study by Kareev et al. (2002) is especially relevant to our approach because it measured the association not in terms of a difference in means, but in terms of the proportion of participants who believed the large sample to be the more variable one. This characterization is similar to the one adopted in this paper (eq. 1). However, neither previous study actually analyzes sample variability. For example, in Kareev et al. (2002), without the information about the actual samples observed by the participants it is not possible to rule out the possibility that people perceive a large sample population as more variable even if the observed sample is not the more variable one. The study by Linville et al. (1989) is subject to the same limitation. To overcome this limitation, we collected novel evidence.

\section{Experiment 1: Sample Size and Perceived Variability}

Our experiment design was inspired by Experiment 2 in Kareev et al. (2002). The main difference is that we measured the variabilities of the samples observed by each participant. Moreover, the cover story invokes social groups instead of physical objects.

Design. After providing their consent, participants received general instructions: "Imagine you work for the school review board that oversees a specific district of the city. In this quarter, you need to write a report about the performance of the schools that are in your district. The report focuses on the math scores of the pupils. There are two schools in your district: Norester Academy and Oak Mountain High School. In this experiment, you will observe the math scores first from one school and then from the other one. In the end, you will have to judge which school has the larger variety of scores." Participants then observed a random sample of numbers from one school and in the following block a random sample from the other school. One sample size was 5, the other, 50. The pairing of school and sample size was randomized as well as the order in which the two samples were presented. The samples were drawn from the same distribution. We used a symmetrical distribution which ranged from 2 to 9 with the following frequencies: $[0.01,0.06,0.17,0.26,0.26,0.17,0.06,0.01]$. This distribution is a rescaled and discretized beta distribution with parameters $\alpha=\beta=5$. Each participant observed a unique sample from the distribution. Before the first item in each sample, participants saw a fixation cross for 450 milliseconds. Then digits appeared on the screen in quick succession (each digit remained on the screen for 600 milliseconds). This procedure is similar to that described in Goldstein and Rothschild's (2014) paper on perception of distributions.

After participants observed the samples from the two schools, they answered three questions pertaining to the perceived variabilities of the math scores.

Q1. This question was incentivized. Participants were told: "Suppose that two students are randomly chosen from one of the two schools. Let us call A and B their math scores. Let $\mathrm{D}$ be the difference between these two numbers. You will get a bonus of $\mathrm{D}$ points. That is, the larger the difference between the two numbers, the higher your bonus (the bonus cannot be negative)." Then participants faced a choice between the two schools. The goal was thus to select the school with the higher variability since doing so would increase the probability to receive a higher payoff. At the end of the experiment, two numbers were randomly drawn from the chosen school and participants were paid a bonus proportional to $\mathrm{D}$.

Q2. Participants were presented with a continuous slider and asked to indicate which school had the larger "variety of math scores." The minimal value of the slider was -100 (e.g., "Norester Academy has more variety"). The maximal value was 100 (e.g.,"Oak Mountain High School has more variety") and had a midpoint at 0 (e.g., "The schools have the same variety"). (The schools at the end of the scales were randomized, and the numeric values were not shown to the participants).

Q3. Participants were asked to imagine two pupils and their scores from each of the two schools. They were then asked to indicate the school for which they predicted the two numbers to be closer to each other.

The experiment was administered as a Qualtrics online survey to 652 participants recruited via Amazon Mechanical Turk. Participants received a fixed payment for their time and a bonus proportional to D in Q1. ${ }^{16}$

\section{Prediction and background conditions.}

Main prediction: Asymmetry in perceived variabilities. Most participants will perceive the large sample school as the more variable. This prediction of our model was contingent on the following two background conditions:

Background condition 1: Asymmetry in sample variabilities. Consistent with the simulations reported earlier in the paper, we anticipated that for most participants the sample variance of the large sample school $\left(V_{L}^{c}\right)$ would be larger than the sample variance of the small sample school $\left(V_{S}^{c}\right)$ : $P\left(V_{L}^{c}>V_{S}^{c}\right)>.5$.

Background Condition 2: Effect of sample variability on perceived variability. The proportion of participants choosing the large sample school will be higher when the large sample school has the higher variability than when it has the lower variability.

Results. Because all three questions asked people to indicate which school had the higher variability, we combined the responses to the three questions in an aggregate score.

\footnotetext{
${ }^{16}$ Experimental data will be made available on Open Science Framework upon publication of this paper.
} 
Participants who indicated that Norester Academy was the more variable in the majority (minority) of the responses to Q1, Q2, and Q3 were coded as perceiving Norester Academy as the more (less) variable school. ${ }^{17}$ Results for each question are reported in Table B1 in the Appendix.

The results were consistent with our predictions. We report our analyses using the corrected sample variance as the estimator of sample variability. Similar results held with the other estimators discussed above.

Background condition 1: Asymmetry in sample variabilities. For more than half of the participants, the sample variability of the large sample school was the larger one: $V_{S}^{c}$ : $P\left(V_{L}^{c}>V_{S}^{c}\right)=.56,95 \% C I=[.53, .60]$.

Background condition 2: Effect of sample variability on perceived variability. We computed the proportion of participants who perceived the large sample school as the more variable school when its sample variance was larger. It was equal to $.63(95 \% C I=[.58, .68], n=367)$. The corresponding proportion conditional on the larger school having the lower sample variance was $.50(95 \% C I=[.44, .56], n=283)$. The difference was clearly positive $(d=0.13 ; 95 \% C I=$ $[0.05,0.21])$. This indicates that participants' responses were sensitive to the difference in sample variability.

Test of the main prediction: Asymmetry in perceived variabilities. Most participants perceived the large sample school as more variable than the small sample school: The proportion was $58 \%(95 \% C I=[.51, .61])$.

Discussion. The two background conditions were satisfied, as was our main prediction: Most participants perceived the large sample school as more variable than the small sample school even though the samples were generated from the same underlying distribution. Sample size had a positive effect on sample variability, and the difference in sample variabilities had a positive effect on the difference in perceived variabilities. The tendency to perceive the large sample school as the more variable school is thus at least partly explained by the difference in sample variabilities.

In this experiment, there was no notion of in-group or out-group. Therefore, these results only provide indirect evidence that our sample-based explanation could explain the in-group heterogeneity effect. In Experiment 2, we addressed this shortcoming by instructing people to imagine they are part of a social group.

\section{Experiment 2: Sample Asymmetry, In-Group Hetero- geneity Effect, and In-Group Homogeneity Effect}

Experiment 2 replicated the design of Experiment 1 except for the fact that the participants were instructed to imagine they were an alumnus of one of the two schools. This school was thus the in-group; the other was the out-group. We manipulated the relative sizes of the samples obtained about the in-group and the out-group to demonstrate that the asymmetry in sample sizes could produce an in-group heterogeneity effect or its opposite, an in-group homogeneity effect.

Design. After the general instructions, the participants were told that last week they received an email from the alumni association of their school (they were randomly assigned to either Norester Academy or Oak Mountain High School) inviting them to the upcoming homecoming celebrations. Participants were randomly assigned to two experimental conditions. In the large in-group sample condition they obtained a larger sample about their in-group (their school). In the small in-ground sample condition they obtained a larger sample of the out-group (the other school). The rest of the design was exactly the same as in Experiment 1 .

We recruited 1,408 participants using Amazon Mechanical Turk.

Prediction and background conditions.

Main prediction: Asymmetry in perceived variabilities. In the large in-group sample condition, there will be an ingroup heterogeneity effect: Most participants will perceive the in-group as more variable than the out-group. In the small in-group sample condition, there will be an opposite asymmetry: an in-group homogeneity effect. As in experiment 1 , these predictions about perceived group variability were contingent on two background conditions:

Background condition 1: Asymmetry in sample variabilities. In the large in-group sample condition, the sample variability of the in-group will be the larger for most participants: $P\left(V_{\text {in }}^{c}>V_{\text {out }}^{c}\right)>$.5. In the small in-group sample condition, the opposite will hold: $P\left(V_{\text {in }}^{c}>V_{\text {out }}^{c}\right)<.5$.

Background condition 2: Effect of sample variability on perceived variability. The proportion of participants who perceive the large sample school as the more variable will be higher when the large sample school has the higher variability than when it has the lower variability.

Results. To measure participants' perceptions of relative group variability, we used the same aggregate score as in Experiment $1 .{ }^{18}$ Results for each question are reported in Table B2 of the Appendix.

Background condition 1: Asymmetry in sample variabilities. In the large in-group sample condition, the sample variability of the in-group was the larger one for most participants $(p=.56,95 \% C I=[.52, .59])$. In the small in-group

\footnotetext{
${ }^{17}$ Two participants responded 0 to Q2 and indicated two different schools in their responses to Q1 and Q3. The responses provided by these participants did not favor one school or the other and thus they were dropped from our analyses. This led to a sample size of 650 participants.

${ }^{18}$ Five participants responded 0 to Q2 and indicated two different schools in their responses to Q1 and Q3. The responses provided by these participants do not favor one school or the other and thus were dropped from our analyses, bringing the size of the sample of participants to 1,403 .
} 
sample condition, the sample variability of the in-group was larger for less than half of the participants $(p=.42,95 \% C I=$ $[.38, .46])$.

Background condition 2: Effect of sample variability on perceived variability. We computed the proportion of participants who perceived the large sample school as the more variable when its sample variance was the larger one. It was equal to $.65(95 \% C I=[.62, .69], n=799)$. The corresponding proportion conditional on the larger school having the lower sample variance was .56 $(95 \% C I=[.52, .60], n=604)$. This positive difference $(d=0.09 ; 95 \% C I=[.04, .14])$ replicated the evidence from Experiment 1 that participants were sensitive to the difference in sample variability.

Main prediction: Asymmetry in perceived variabilities. The results are consistent with our predictions. In the large in-group sample condition, there was an in-group heterogeneity effect: Most participants perceived the in-group as the more variable group $(p=.62,95 \% C I=[.59, .66])$. By contrast, in the small in-group sample condition there was an in-group homogeneity effect: Less than half of the participants perceived the in-group as the more variable group $(p=.39,95 \% C I=[.36, .43])$. In other words, most participants perceived the out-group as the more variable group.

Discussion. The results of Experiment 2 are consistent with the predictions of our sampling mechanism. When participants obtained a larger sample about their in-group, an ingroup heterogeneity effect emerged. When they obtained a smaller sample about their in-group, an in-group homogeneity effect emerged. These results provide evidence for the causal effect of the difference in sample sizes on the nature of the perceived asymmetry in group variabilities. Moreover, they are consistent not only with existing findings on the ingroup heterogeneity effect (as discussed at the beginning of this section) but also with findings on perceived in-group homogeneity. For example, B. Simon and Brown (1987) assigned participants to groups of different perceived size (minority vs. majority) and asked them to judge the groups on several dimensions (see also B. Simon \& Pettigrew, 1990b). Similar to our results, they found that minority group members perceived the in-group as more homogeneous than the out-group. Their explanation differs from ours, however, as it relies on social identity and motivated reasoning.

The in-group/out-group manipulation implemented in Experiment 2 was fairly minimal since participants were simply asked to imagine they were part of one of the groups. A more thorough investigation of how our sampling-based mechanism and processes that rely on motivated cognition interact in explaining asymmetries in perceived group heterogeneity would require stronger manipulations of group identity (e.g., random assignment to groups of participants in a behavioral laboratory). This is an interesting avenue for future research.

\section{Implications for Social Cognition}

\section{Use of stereotypes}

Our model has implications for the conditions under which people are more likely to make stereotype-based inferences about members of social groups. This is because people are less likely to rely on stereotypes if they perceive the group as more variable. For example, Ryan et al. (1996) measured perceived out-group variability using the percentage task (a variation of the "proportion of members who possess a trait" measure) and the range task (a variation of the "range" measure). They then asked the participants to indicate the extent to which specific members of the outgroup possessed a stereotypical trait. The results of three experiments show that the more variable the participants perceived the out-group to be, the less stereotypical their judgments about specific members were. In another study, making people more aware of the out-group variability led to more prosocial behavior towards individual out-group members (Brauer \& Er-Rafiy, 2011). Together with these findings, our results imply that people are more likely to rely on stereotypes when judging members of the out-group than when judging members of the in-group.

This casts new light on the benefit of intergroup contact. Research has shown that stereotypes about out-groups are frequently negative (Brewer, 1999; Hewstone, Rubin, \& Willis, 2002). These are harmful to social harmony: People relying on negative stereotypes are more likely to interpret ambiguous information negatively and less likely to gather additional information about the out-group. It has been proposed that negative attitudes and stereotypes toward out-groups are often the results of adaptive sampling (the tendency to sample again alternatives that led to positive outcomes and to avoid alternatives that lead to negative outcomes (e.g., Denrell, 2005). This leads people to obtain samples about the out-group that are negatively biased. Additional sampling of the out-group has the potential to correct for this bias in attitudes toward out-groups by providing information about out-groups that is more positive overall due to the regression-to-the-mean phenomenon (Denrell, 2005; Fazio, Eiser, \& Shook, 2004; Le Mens \& Denrell, 2011). Our results suggest another benefit of additional sampling of the out-group: a lower propensity to use stereotypes. Because our mechanism does not rely on hedonic sampling, this implies that additional contact could help foster social harmony even in settings where attitudes about the groups are not the results of hedonic sampling. In other words, our mechanism expands the scope of sampling explanations for the benefits of intergroup contact.

\section{Perception of group complexity}

Our sampling approach makes a simple but powerful assumption: The focal agent is more likely to sample from the 
in-group than from the out-group. This paper focused on just one of the consequences of this asymmetry in perceived group variabilities; another possible consequence concerns the complexity of the mental representations of the groups. Evidence gathered by Linville (1982) suggests that people have more complex cognitive representations of in-groups than of out-groups. A sampling asymmetry can help explain this result, since one measure of the complexity of the representation of the group is the number of features used to characterize its members. The larger the number of unique features a person invokes, the more complex their representation of a group (Linville, 1982; Quattrone \& Jones, 1980). An in-group sampling advantage means that an individual encounters a larger set of unique features for the in-group than for the out-group, leading them to perceive the in-group as more complex than the out-group.

A simple model can illustrate this mechanism. Let us assume that each group member is represented by several binary features (they either have them or not). For each group member, a set of unique features is drawn from a large set of features. As in the previous models, the agent samples from the in-group with a probability $r>0.5$. After sampling for $t$ periods, the agent has collected samples of unique features that characterize members of the in-group and out-group. Ancillary computer simulations of this model showed that the probability that the in-group is characterized by a larger number of features than the out-group is above 0.5. This illustration is only one way of formalizing the relationship between sample size and perceived group complexity. A more comprehensive analysis of this relationship is an exciting direction for future research.

\section{Extremity of judgments about group members}

Our argument in this paper exploits the positive association between sample size and variability to explain the asymmetry in perception of group variability. Another interesting implication of this positive association pertains to the impact of experience on judgment extremity. Several studies have found that participants evaluated out-group members more extremely than in-group members (Linville \& Jones, 1980; Marques \& Paez, 1994; Marques, Yzerbyt, \& Leyens, 1988; Pinto, Marques, Levine, \& Abrams, 2010). For example, Linville and Jones (1980) asked white participants to evaluate law school applications that mentioned the race of the applicant. The results showed that a black applicant with strong credentials was judged more favorably than an otherwise identical white applicant. Again, the sampling asymmetry can provide some insight into this finding.

To illustrate the link between experience and extremity, let us define an extreme observation as one drawn from the tail of the perceived distribution. More sampling leads not only to higher sampled variability but also to fatter tails of the sampled distribution. This implies that with larger samples it will become increasingly hard for something to be perceived as extreme, because the requirement for being perceived as extreme becomes more stringent: The threshold for "extremity" is pushed further away from the mean. This implies that extreme judgments would be less likely in later periods.

Consider the following simple model as an illustration. For 100 periods, an individual samples from a group where all members have one feature that is distributed according to a standard normal distribution, $N(0,1)$. Let us assume that every new observation is classified into three categories: "within expectations," "great," or "terrible." The boundaries of the expectations are defined by the range of the observed sample up to time $t$. A member is classified as "great" only if the feature value is above the higher boundary of their expectations. Similarly, a member is classified as "terrible" if the feature value is below the lower boundary of the agent's expectations. Computer simulations of this model showed that the probability that a new member is classified as "great" was higher after the first 10 periods than after 90 periods. This example illustrates an important implication of experience sampling: It changes reference intervals and thus perceptions of extremity. A more comprehensive analysis of how sampling affects judgment extremity is an interesting avenue for future research.

\section{The polarization of attitudes across social groups}

Consider the standard setting studied in investigations of group polarization in which individuals who hold an opinion ("for" or "against") about an issue join a group discussion about this issue (Isenberg, 1986; McGarty, Turner, Hogg, David, \& Wetherell, 1992; Moscovici \& Zavalloni, 1969). After the discussion, the opinions of group members tend to become more extreme in the direction that was prevailing in the group before the discussion. One of the main explanations for such "group polarization" is the Persuasive Argument Theory (Burnstein \& Vinokur, 1973, 1977). Suppose most group members hold a "for" stance on the issue. Persuasive Argument Theory contends that those who take part in the discussion are exposed to arguments in favor of the issue they had not thought about themselves (described as "rare" or "novel" arguments). Opinion extremization ensues. 19

The essence of this explanation is similar to the core idea of our model: exposure to a larger sample of instances leads to an increase in the diversity of sampled instances. Two distinct measures of diversity we have used in an earlier section are relevant to this setting. The first one is the number of

\footnotetext{
${ }^{19}$ According to Persuasive Argument Theory, arguments are considered persuasive if they are valid and novel (i.e., they add to the diversity of arguments the agent has about the issue). If the arguments that are presented are valid and novel, the individuals will shift their attitudes more than when they are valid and not novel (Kaplan, 1977; Vinokur \& Burnstein, 1978).
} 
subgroups "hit" by the sample of observations in the subsection Number of Subgroups (p. 12). Applied to the persuasive argument setting, this implies that the number of unique arguments sampled by the individual increases with sample size. The second relevant measure of diversity is the range (p. 11). Applied to the persuasive argument setting, this implies that the strength of the strongest argument to which the individual has been exposed increases with sample size.

Adapting our sampling approach to the one-group setting described above is straightforward. The focal agent interacts with members of the group. Because the "for" stance dominates in the group, they tend to hear a larger sample of arguments supporting the "for" stance than the "against" stance. This implies, in turn, that the agent will hear a larger number of unique arguments in support of the "for" stance. Moreover, there will be an asymmetry in the maximal strength of sampled arguments: the strongest arguments the agent hears are more likely to be in support of the "for" stance than in support of the "against" stance.

This reasoning can also be applied to a two-group setting. Consider a population made of two groups $G_{1}$ and $G_{2}$. Most members of $G_{1}$ are in favor of the issue, whereas most members of $G_{2}$ are against the issue. Suppose a new person joins group $G_{1}$. She chooses whether she should support the issue or not. She already has some arguments supporting the "for" and "against" stances. She makes her decision based on the arguments she hears from other people about the issue, and she adopts the stance about which she hears more novel and stronger arguments (Chaiken, 1980; Mackie, Worth, \& Asuncion, 1990). Once she adopts a stance, she develops a new argument supporting this stance (with some probability). A similar process applies to the new members of $G_{2}$. Ancillary simulations indicate that such process can lead to opinion polarization across groups: more and more people support the issue in $G_{1}$ whereas fewer and fewer people do so in $G_{2}$. This is because more unique and stronger arguments supporting the issue prevail in $G_{1}$ whereas more unique and stronger arguments against the issue prevail in $G_{2}$.

This example illustrates that our sampling mechanism can extend the scope of the Persuasive Argument Theory: it specifies how this theory can contribute to explaining the polarization of opinions not only within a group but also across social groups. Crucial to the mechanism is the positive feedback loop (or "rich-gets-richer" dynamic) that makes the dominant stance in a group more and more dominant as new members join the group. This occurs because a new group member has a higher probability to hear unique and stronger argument in favor of the issue dominant in her group.

\section{Biases in the Perceived Variability of Probability Distributions}

The focus of this paper lies on the perception of relative group variabilities. Yet, on an abstract level, our model is about the perception of the variability of probability distributions. In the introduction of the paper we noted that the distribution of corrected sample variance is skewed in such a way that most of the time, sample variance is lower than true variance. Here we return to this observation and explore basic empirical predictions that derive from it: When people attempt to estimate the variability of a population on a feature, subjective variability will more often than not be smaller than population variability. ${ }^{20}$ This is because subjective variability itself depends on sample variability and sample variability tends to underestimate population variability.

To test this prediction, we analyzed data from the LISS panel discussed in p. 9. This survey assessed participants' beliefs about the distributions of 10 characteristics in the population of the Netherlands. It also assessed their beliefs about the distributions of these characteristics in their social circles. Like the authors of the original study, we took the reported social circle distribution as a proxy for the distribution a participant sampled from the environment (Galesic et al., 2012).

Participants also reported where they stand on these 10 characteristics (on the same seven-level scale as the one used to elicit the distributions). Because the set of participants makes a representative sample of the population of the Netherlands, it is possible to use the survey responses to construct the distributions of these 10 features in the national population. $^{21,22}$

For each of the 10 features, we denote by $V_{P}$ the variance of the national population. We denote by $V_{S C}$ the variance of the reported social circle distribution and by $V_{\hat{P}}$ the variance of the subjective population distribution. Our sampling approach predicts that the variance of the subjective population distribution will tend to be lower than the variance of the population distribution: $P\left(V_{\hat{P}}<V_{P}\right)>$.5. Empirical estimations reported in Table 1 are consistent with this prediction. The

\footnotetext{
${ }^{20}$ Suppose the relevant measure of variability is variance $\sigma^{2}$ and the sample based estimator of variance is the corrected sample variance $V^{c}$. We have$$
P\left(V^{c}<\sigma^{2}\right)>.5
$$

${ }^{21}$ The data were collected in two waves. In the first wave participants reported the perceived population distribution and in the second wave participants reported the social circle information. In each wave, participants indicated their position in the distribution. The results discussed in the text are based on a real distribution constructed from the responses (of participants about their position) collected in the first wave. The results are essentially the same for the distribution based on the second-wave responses, so we do not report these additional analyses here.

${ }^{22}$ There is some irony in calling a distribution constructed on the basis of a sample of a smaller size than the true population (the population of the Netherlands) the "population distribution." But because this sample is large (about 1,400 people), its sample variance is very likely to be almost identical to the population variance.
} 
propensity for underestimation of the variance was higher than $50 \%$ for most characteristics. In the pooled data, the proportion of underestimation was $61 \%$. In summary, there was a general tendency toward underestimating the variance of the population distributions.

A similar pattern characterized social circle distributions. For most participants, the variance of the social circle distribution was lower than the variance of the true population distribution. This was the case for all 10 characteristics, as well as for the pooled data (Table 1 , column $P\left(V_{S C}<V_{P}\right)$ ).

Finally, these data provide evidence that the propensity for underestimating variance depends on the social circle distribution. We computed two conditional probabilities: the probability that participants underestimate the variance of the population distribution when their social circle underestimates it, $P\left(V_{\hat{P}}<V_{P} \mid V_{S C}<V_{P}\right)$, and when it overestimates it, $P\left(V_{\hat{P}}<V_{P} \mid V_{S C}>V_{P}\right)$. If the variance of the subjective distribution depends on the sample distribution, the first of these two quantities should be the larger one. This prediction is borne out in the data. In the pooled data across all characteristics $P\left(V_{\hat{P}}<V_{P} \mid V_{S C}<V_{P}\right)$ was 0.64 whereas $P\left(V_{\hat{P}}<V_{P} \mid V_{S C}>V_{P}\right)$ was only 0.39 . This asymmetry held for all 10 characteristics (Table 1).

Overall, these analyses of the LISS panel data provide evidence consistent with our prediction that subjective variability will tend to underestimate population variability and that this tendency is at least partially driven by the fact that sample variability tends to be smaller than population variability.

\section{Conclusion}

People frequently obtain larger samples of information about in-groups than about out-groups. Because estimators of variability tend to be right-skewed when samples are not very large, people will tend to perceive in-groups as more variable than out-groups. In this paper, we showed that this in-group heterogeneity effect emerges under a wide range of assumptions about how people process information. In particular, it emerges even when people process information rationally. This implies that sampling constraints imposed by the environment are sufficient to imply the emergence of the in-group heterogeneity effect.

The asymmetry in perceived variability we discussed in this paper is not limited to judgments about social groups. Assessments of variability are essential in a variety of domains such as risk perception (Hertwig, Barron, Weber, \& Erev, 2004; Slovic, 2000; Weber et al., 2004), confidence in judgment (Einhorn \& Hogarth, 1978; Juslin et al., 2007), categorization (Hahn, Bailey, \& Elvin, 2005; Park, Judd, \& Ryan, 1991; N. Stewart \& Chater, 2002) and category-based generalization (Chater \& Vitányi, 2003; Hahn et al., 2005). Exploring how the structure of the environment systematically affects assessments of variability in these other domains and the judgments and behavior contingent on these assessments is an exciting avenue for future research.

\section{References}

Alves, H., Koch, A., \& Unkelbach, C. (2016). My friends are all alike - the relation between liking and perceived similarity in person perception. Journal of Experimental Social Psychology, 62, 103-117. doi: 10.1016/j.jesp.2015.10.011

Anderson, J. R. (1991). The adaptive nature of human categorization. Psychological Review, 98(3), 409-429. doi: 10.1037/0033-295X.98.3.409

Badea, C., Brauer, M., \& Rubin, M. (2012). The effects of winning and losing on perceived group variability. Journal of Experimental Social Psychology, 48(5), 1094-1099. doi: 10.1016/j.jesp.2012.03.006

Beach, L. R., \& Scopp, T. S. (1968). Intuitive statistical inferences about variances. Organizational Behavior and Human Performance, 3(2), 109-123. doi: 10.1016/00305073(68)90001-9

Boldry, J. G., \& Gaertner, L. (2006). Separating status from power as an antecedent of intergroup perception. Group Processes \& Intergroup Relations, 9(3), 377-400. doi: 10.1177/1368430206064640

Boldry, J. G., Gaertner, L., \& Quinn, J. (2007). Measuring the measures a meta-analytic investigation of the measures of outgroup homogeneity. Group Processes \& Intergroup Relations, 10(2), 157-178. doi: $10.1177 / 1368430207075153$

Brauer, M., \& Er-Rafiy, A. (2011). Increasing perceived variability reduces prejudice and discrimination. Journal of Experimental Social Psychology, 47(5), 871-881. doi: 10.1016/j.jesp.2011.03.003

Brewer, M. B. (1999). The psychology of prejudice: Ingroup love and outgroup hate? Journal of Social Issues, 55(3), 429-444. doi: 10.1111/0022-4537.00126

Brighton, H., \& Gigerenzer, G. (2012). Homo Heuristicus and the BiasâVariance Dilemma. In Action, perception and the brain (pp. 68-91). London: Palgrave Macmillan UK. doi: $10.1057 / 9780230360792_{4}$

Brown, R. (2000). Social identity theory: Past achievements, current problems, and future challenges. European journal of social psychology, 30(6), 745-778. doi: 10.1002/10990992(200011/12)30:6<745::AID-EJSP24>3.0.CO;2-O

Brunswik, E. (1952). The conceptual framework of psychology. Chicago: The University of Chicago Press.

Burnstein, E., \& Vinokur, A. (1973). Testing two classes of theories about group induced shifts in individual choice. Journal of Experimental Social Psychology, 9(2), 123137.

Burnstein, E., \& Vinokur, A. (1977). Persuasive argumentation and social comparison as determinants of attitude 
Table 1

Comparison Between the Variance of the Perceived Population Distribution $\left(V_{\hat{P}}\right)$, the Variance of the Social Circle Distribution $\left(V_{S C}\right)$, and the Variance of the Real Population Distribution $\left(V_{P}\right)$ in LISS Panel Data.

\begin{tabular}{c|c|c|c|c|c}
\hline & $P\left(V_{\hat{P}}<V_{P}\right)$ & $P\left(V_{S C}<V_{P}\right)$ & $P\left(V_{\hat{P}}<V_{P} \mid V_{S C}<V_{P}\right)$ & $P\left(V_{\hat{P}}<V_{P} \mid V_{S C}>V_{P}\right)$ & Difference \\
\hline Amount of stress & .87 & .96 & .88 & .80 & $.08[-.03, .20]$ \\
Personal income & .52 & .90 & .53 & .37 & $.16[.07, .25]$ \\
Household income & .72 & .94 & .73 & .59 & $.15[.03, .26]$ \\
Wealth & .70 & .94 & .71 & .56 & $.14[.03, .26]$ \\
Number of friends & .67 & .93 & .68 & .47 & $.21[.10, .32]$ \\
Level of education & .26 & .87 & .28 & .15 & $.13[.06, .18]$ \\
Number of problems & .72 & .92 & .74 & .59 & $.20[.09, .30]$ \\
Number of meetings & .78 & .93 & .79 & .17 & $.20[.09, .30]$ \\
Number of conflicts & .33 & .88 & .35 & .07 & $.18[.11, .26]$ \\
Number of dates & .20 & .74 & .24 & .39 & $.17[.08, .27]$ \\
\hline All Data & .61 & .92 & .64 & $.25[0.22,0.28]$ \\
\hline
\end{tabular}

Note: $95 \%$ confidence intervals are in brackets.

polarization. Journal of Experimental Social Psychology, 13(4), 315-332.

Campbell, D. T. (1958). Common fate, similarity, and other indices of the status of aggregates of persons as social entities. Behavioral Science, 3(1), 14-25. doi: 10.1002/bs.3830030103

Casella, G., \& Berger, R. L. (2002). Statistical inference, 2nd edition. Pacific Grove, CA: Duxbury.

Chaiken, S. (1980). Heuristic versus systematic information processing and the use of source versus message cues in persuasion. Journal of Personality and Social Psychology, 39(5), 752-766. doi: 10.1037/0022-3514.39.5.752

Chaiken, S. (1987). The heuristic model of persuasion. In M. P. Zanna, J. M. Olson, \& C. P. Herman (Eds.), Social influence: The Ontario symposium, volume 5 (pp. 3-39).

Chater, N., \& Vitányi, P. M. B. (2003). The generalized universal law of generalization. Journal of Mathematical Psychology, 47(3), 346-369. doi: 10.1016/S00222496(03)00013-0

Davis, J. A., Smith, T. W., \& Marsden, P. V. (2016). General social survey, 1993, 1998, 2000, 2002 with cultural, information security, and freedom modules [United States]. Inter-university Consortium for Political and Social Research [distributor]. doi: 10.3886/ICPSR35536.v2

Denrell, J. (2005). Why most people disapprove of me: Experience sampling in impression formation. Psychological Review, 112(4), 951-978. doi: 10.1037/0033295X.112.4.951

Einhorn, H. J., \& Hogarth, R. M. (1978). Confidence in judgment: Persistence of the illusion of validity. Psychological Review, 85(5), 395-416.

Fazio, R. H., Eiser, J. R., \& Shook, N. J. (2004). Attitude formation through exploration: Valence asymmetries. Journal of Personality and Social Psychology, 87(3), 293-311. doi: 10.1037/0022-3514.87.3.293
Fiedler, K. (1996). Explaining and simulating judgment biases as an aggregation phenomenon in probabilistic, multiple-cue environments. Psychological Review, 103(1), 193.

Fiedler, K. (2007). Construal level theory as an integrative framework for behavioral decision-making research and consumer psychology. Journal of Consumer Psychology, 17(2), 101-106. doi: 10.1016/S1057-7408(07)70015-3

Fiedler, K. (2012). Meta-cognitive myopia and the dilemmas of inductive-statistical inference. In B. H. Ross (Ed.), The psychology of learning and motivation, Vol. 57 (pp. 1-55).

Fiedler, K., \& Juslin, P. (2006). Information sampling and adaptive cognition. Cambridge, United Kingdom: Cambridge University Press.

Fiedler, K., Kemmelmeier, M., \& Freytag, P. (1999). Explaining Asymmetric Intergroup Judgments through Differential Aggregation: Computer Simulations and Some New Evidence. European Review of Social Psychology, 10(1), 1-40. doi: 10.1080/14792779943000008

Galesic, M., Olsson, H., \& Rieskamp, J. (2012). Social sampling explains apparent biases in judgments of social environments. Psychological Science, 23(12), 1515-1523. doi: $10.1177 / 0956797612445313$

Goldstein, D. G., \& Rothschild, D. (2014). Lay understanding of probability distributions. Judgment and Decision Making, 9(1), 1-14.

Hahn, U., Bailey, T. M., \& Elvin, L. B. C. (2005). Effects of category diversity on learning, memory, and generalization. Memory \& Cognition, 33(2), 289-302. doi: 10.3758/BF03195318

Haslam, N. (2006). Dehumanization: An integrative review. Personality and social psychology review, 10(3), 252-264.

Haslam, S. A., Oakes, P. J., Turner, J. C., \& McGarty, C. (1995). Social categorization and group homogeneity: Changes in the perceived applicability of 
stereotype content as a function of comparative context and trait favourableness. British Journal of Social Psychology, 34(2), 139-160. doi: 10.1111/j.20448309.1995.tb01054.x

Hertwig, R., Barron, G., Weber, E. U., \& Erev, I. (2004). Decisions from experience and the effect of rare events in risky choice. Psychological Science, 15(8), 534-539. doi: 10.1111/j.0956-7976.2004.00715.x

Hewstone, M., Rubin, M., \& Willis, H. (2002). Intergroup bias. Annual Review of Psychology, 53, 575-604. doi: 10.1146/annurev.psych.53.100901.135109

Hogarth, R. M., \& Einhorn, H. J. (1992). Order effects in belief updating: The belief-adjustment model. Cognitive psychology, 24(1), 1-55. doi: 10.1016/0010-0285(92)90002$\mathrm{J}$

Isenberg, D. J. (1986). Group polarization: A critical review and meta-analysis. Journal of personality and social psychology, 50(6), 1141.

Jones, E. E., Wood, G. C., \& Quattrone, G. A. (1981). Perceived variability of personal characteristics in in-groups and out-groups: The role of knowledge and evaluation. Personality and Social Psychology Bulletin, 7(3), 523528. doi: $10.1177 / 014616728173024$

Judd, C. M., \& Park, B. (1988). Out-group homogeneity: Judgments of variability at the individual and group levels. Journal of Personality and Social Psychology, 54(5), 778 788. doi: 10.1037/0022-3514.54.5.778

Judd, C. M., Ryan, C. S., \& Park, B. (1991). Accuracy in the judgment of in-group and out-group variability. Journal of Personality and Social Psychology, 61(3), 366-379. doi: 10.1037/0022-3514.61.3.366

Juslin, P., Winman, A., \& Hansson, P. (2007). The naïve intuitive statistician: A naïve sampling model of intuitive confidence intervals. Psychological Review, 114(3), 678703. doi: 10.1037/0033-295X.114.3.678

Kaplan, M. F. (1977). Discussion polarization effects in a modified jury decision paradigm: Informational influences. Sociometry, 262-271.

Kareev, Y., Arnon, S., \& Horwitz-Zeliger, R. (2002). On the misperception of variability. Journal of Experimental Psychology: General, 131(2), 287-297. doi: 10.1037/00963445.131.2.287

Konovalova, E., \& Le Mens, G. (2018). Learning variability from experience. In Proceedings of the 40th annual conference of the cognitive science society (pp. 1942-1947). Austin, TX: Cognitive Science Society.

Kunda, Z. (1990). The case for motivated reasoning. Psychological Bulletin, 108(3), 480-498. doi: 10.1037/00332909.108.3.480

Le Mens, G., \& Denrell, J. (2011). Rational learning and information sampling: On the "naivety" assumption in sampling explanations of judgment biases. Psychological Review, 118(2), 379-392. doi: 10.1037/a0023010
Liberman, N., Trope, Y., \& Wakslak, C. (2007). Construal level theory and consumer behavior. Journal of Consumer Psychology, 17(2), 113-117. doi: 10.1016/S10577408(07)70017-7

Linville, P. W. (1982). The complexity-extremity effect and age-based stereotyping. Journal of Personality and Social Psychology, 42(2), 193-211. doi: 10.1037/00223514.42.2.193

Linville, P. W., \& Fischer, G. W. (1998). Group variability and covariation: Effects on intergroup judgment and behavior. In C. Sedikides, J. Schopler, \& C. A. Insko (Eds.), Intergroup cognition and intergroup behavior (pp. 123150). Mahwah, NJ, US: Lawrence Erlbaum Associates Publishers.

Linville, P. W., Fischer, G. W., \& Salovey, P. (1989). Perceived distributions of the characteristics of in-group and out-group members: Empirical evidence and a computer simulation. Journal of Personality and Social Psychology, 57(2), 165-188. doi: 10.1037/0022-3514.57.2.165

Linville, P. W., Fischer, G. W., \& Yoon, C. (1996). Perceived covariation among the features of ingroup and outgroup members: The outgroup covariation effect. Journal of Personality and Social Psychology, 70(3), 421-436. doi: 10.1037/0022-3514.70.3.421

Linville, P. W., \& Jones, E. E. (1980). Polarized appraisals of out-group members. Journal of Personality and Social Psychology, 38(5), 689-703. doi: 10.1037/00223514.38.5.689

Lorenzi-Cioldi, F. (1998). Group status and perceptions of homogeneity. European Review of Social Psychology, 9(1), 31-75. doi: 10.1080/14792779843000045

Mackie, D. M., Worth, L. T., \& Asuncion, A. G. (1990). Processing of persuasive in-group messages. Journal of Personality and Social Psychology, 58(5), 812-822. doi: 10.1037/0022-3514.58.5.812

March, J. G. (1996). Learning to be risk averse. Psychological Review, 103(2), 309-319. doi: 10.1037/0033295X.103.2.309

Marques, J. M., \& Paez, D. (1994). The 'black sheep effect': Social categorization, rejection of ingroup deviates, and perception of group variability. European Review of Social Psychology, 5(1), 37-68. doi: 10.1080/14792779543000011

Marques, J. M., Yzerbyt, V. Y., \& Leyens, J.-P. (1988). The 'black sheep effect': Extremity of judgments towards ingroup members as a function of group identification. $E$ ropean Journal of Social Psychology, 18(1), 1-16. doi: 10.1002/ejsp.2420180102

Marsden, P. V. (1987). Core discussion networks of Americans. American Sociological Review, 52(1), 122-131. doi: $10.2307 / 2095397$

Massey, D. S., \& Denton, N. A. (1989). Hypersegregation in us metropolitan areas: Black and Hispanic segregation 
along five dimensions. Demography, 26(3), 373-391. doi: $10.2307 / 2061599$

McGarty, C., Turner, J. C., Hogg, M. A., David, B., \& Wetherell, M. S. (1992). Group polarization as conformity to the prototypical group member. British Journal of Social Psychology, 31(1), 1-19.

McPherson, J. M., \& Smith-Lovin, L. (1987). Homophily in voluntary organizations: Status distance and the composition of face-to-face groups. American Sociological Review, 370-379. doi: 10.2307/2095356

McPherson, M., Smith-Lovin, L., \& Brashears, M. E. (2006). Social isolation in America: Changes in core discussion networks over two decades. American Sociological Review, 71(3), 353-375. doi: 10.1177/000312240607100301

McPherson, M., Smith-Lovin, L., \& Cook, J. M. (2001). Birds of a feather: Homophily in social networks. Annual Review of Sociology, 27, 415-444. doi: 10.1146/annurev.soc.27.1.415

Miller, G. A. (1956). The magical number seven, plus or minus two: Some limits on our capacity for processing information. Psychological Review, 63(2), 81-97. doi: $10.1037 / \mathrm{h} 0043158$

Moscovici, S., \& Zavalloni, M. (1969). The group as a polarizer of attitudes. Journal of personality and social psychology, 12(2), 125.

Niv, Y., Daniel, R., Geana, A., Gershman, S. J., Leong, Y. C., Radulescu, A., \& Wilson, R. C. (2015). Reinforcement learning in multidimensional environments relies on attention mechanisms. The Journal of neuroscience : the official journal of the Society for Neuroscience, 35(21), 814557. doi: 10.1523/JNEUROSCI.2978-14.2015

Ostrom, T. M., Carpenter, S. L., Sedikides, C., \& Li, F. (1993). Differential processing of in-group and out-group information. Journal of Personality and Social Psychology, 64(1), 21-34. doi: 10.1037/0022-3514.64.1.21

Ostrom, T. M., \& Sedikides, C. (1992). Out-group homogeneity effects in natural and minimal groups. Psychological Bulletin, 112(3), 536-552. doi: 10.1037/00332909.112.3.536

Pachur, T., Hertwig, R., \& Rieskamp, J. (2013). Intuitive judgments of social statistics: How exhaustive does sampling need to be? Journal of Experimental Social Psychology, 49(6), 1059-1077. doi: 10.1016/j.jesp.2013.07.004

Park, B., \& Hastie, R. (1987). Perception of variability in category development: Instance-versus abstraction-based stereotypes. Journal of Personality and Social Psychology, 53(4), 621-635. doi: 10.1037/0022-3514.53.4.621

Park, B., \& Judd, C. M. (1990). Measures and models of perceived group variability. Journal of Personality and Social Psychology, 59(2), 173-191. doi: 10.1037/00223514.59.2.173

Park, B., Judd, C. M., \& Ryan, C. S. (1991). Social cat- egorization and the representation of variability information. European Review of Social Psychology, 2(1), 211245. doi: 10.1080/14792779143000079

Park, B., \& Rothbart, M. (1982). Perception of out-group homogeneity and levels of social categorization: Memory for the subordinate attributes of in-group and out-group members. Journal of Personality and Social Psychology, 42(6), 1051-1068. doi: 10.1037/0022-3514.42.6.1051

Park, B., Ryan, C. S., \& Judd, C. M. (1992). Role of meaningful subgroups in explaining differences in perceived variability for in-groups and out-groups. Journal of Personality and Social Psychology, 63(4), 553-567. doi: 10.1037/0022-3514.63.4.553

Pickett, C. L., \& Brewer, M. B. (2001). Assimilation and differentiation needs as motivational determinants of perceived in-group and out-group homogeneity. Journal of Experimental Social Psychology, 37(4), 341-348. doi: 10.1006/jesp.2000.1469

Pinto, I. R., Marques, J. M., Levine, J. M., \& Abrams, D. (2010). Membership status and subjective group dynamics: Who triggers the black sheep effect? Journal of Personality and Social Psychology, 99(1), 107-119. doi: 10.1037/a0018187

Pollard, P. (1984). Intuitive judgments of proportions, means, and variances: A review. Current Psychology, 3(1), 5-18. doi: 10.1007/BF02686528

Quattrone, G. A., \& Jones, E. E. (1980). The perception of variability within in-groups and out-groups: Implications for the law of small numbers. Journal of Personality and Social Psychology, 38(1), 141-152. doi: 10.1037/00223514.38.1.141

Ratcliff, N. J., Hugenberg, K., Shriver, E. R., \& Bernstein, M. J. (2011). The allure of status: High-status targets are privileged in face processing and memory. Personality and Social Psychology Bulletin, 37(8), 1003-1015. doi: 10.1177/0146167211407210

Rubin, M., \& Badea, C. (2007). Why do people perceive ingroup homogeneity on ingroup traits and outgroup homogeneity on outgroup traits? Personality and Social Psychology Bulletin, 33(1), 31-42. doi: 10.1177/0146167206293190

Rubin, M., \& Badea, C. (2012). They're all the same!... but for several different reasons: A review of the multicausal nature of perceived group variability. Current $D i$ rections in Psychological Science, 21(6), 367-372. doi: 10.1177/0963721412457363

Rubin, M., Hewstone, M., \& Voci, A. (2001). Stretching the boundaries: Strategic perceptions of intragroup variability. European Journal of Social Psychology, 31(4), 413-429. doi: 10.1002/ejsp.51

Ryan, C. S., Judd, C. M., \& Park, B. (1996). Effects of racial stereotypes on judgments of individuals: The moderating role of perceived group variability. Journal 
of Experimental Social Psychology, 32(1), 71-103. doi: 10.1006/jesp.1996.0004

Sanborn, A. N., Griffiths, T. L., \& Navarro, D. J. (2010). Rational approximations to rational models: Alternative algorithms for category learning. Psychological Review, 117(4), 1144-1167. doi: 10.1037/a0020511

Shi, L., Griffiths, T. L., Feldman, N. H., \& Sanborn, A. N. (2010). Exemplar models as a mechanism for performing Bayesian inference. Psychonomic Bulletin \& Review, 17(4), 443-464. doi: 10.3758/PBR.17.4.443

Simon, B., \& Brown, R. (1987). Perceived intragroup homogeneity in minority-majority contexts. Journal of Personality and Social Psychology, 53(4), 703. doi: 10.1037/0022-3514.53.4.703

Simon, B., \& Pettigrew, T. F. (1990a). Social identity and perceived group homogeneity evidence for the ingroup homogeneity effect. European Journal of Social Psychology, 20(4), 269-286. doi: 10.1002/ejsp.2420200402

Simon, B., \& Pettigrew, T. F. (1990b). Social identity and perceived group homogeneity: Evidence for the ingroup homogeneity effect. European Journal of Social Psychology, 20(4), 269-286.

Simon, H. A. (1956). Rational choice and the structure of the environment. Psychological Review, 63(2), 129-138. doi: $10.1037 / \mathrm{h} 0042769$

Slovic, P. (2000). Risk, society, and policy series: The perception of risk. London, England: Earthscan Publications.

Soll, J. B. (1999). Intuitive theories of information: Beliefs about the value of redundancy. Cognitive Psychology, 38(2), 317-346.

Stewart, N., \& Chater, N. (2002). The effect of category variability in perceptual categorization. Journal of Experimental Psychology: Learning, Memory, and Cognition, 28(5), 893-907. doi: 10.1037/0278-7393.28.5.893

Stewart, T. L., Vassar, P. M., Sanchez, D. T., \& David, S. E. (2000). Attitude toward women's societal roles moderates the effect of gender cues on target individuation. Journal of Personality and Social Psychology, 79(1), 143-157. doi: 10.1037/0022-3514.79.1.143

Tajfel, H. (1982). Social psychology of intergroup relations. Annual Review of Psychology, 33(1), 1-39.

Trope, Y., \& Liberman, N. (2010). Construal-level theory of psychological distance. Psychological review, 117(2), 440-463. doi: 10.1037/a0018963

van Kempen, R., \& Özüekren, A. Ş. (1998). Ethnic segregation in cities: New forms and explanations in a dynamic world. Urban Studies, 35(10), 1631-1656. doi: 10.1080/0042098984088

Vinokur, A., \& Burnstein, E. (1978). Novel argumentation and attitude change: The case of polarization following group discussion. European Journal of Social Psychology, 8(3), 335-348.

Voci, A., Hewstone, M., Crisp, R. J., \& Rubin, M.
(2008). Majority, minority, and parity: Effects of gender and group size on perceived group variability. Social Psychology Quarterly, 71(2), 114-142. doi: 10.1177/019027250807100203

Weber, E. U., Shafir, S., \& Blais, A.-R. (2004). Predicting risk sensitivity in humans and lower animals: Risk as variance or coefficient of variation. Psychological Review, $111(2), 430-445$.

Yzerbyt, V., Judd, C. M., \& Corneille, O. (2004). The psychology of group perception: Perceived variability, entitativity, and essentialism. New York, NY: Psychology Press.

\section{Appendix A \\ Details of Additional Sensitivity Analyses}

\section{Adaptive Sampling of Groups}

The agent observes two dimensions of the group by sampling it: an attitudinal dimension $A$ and another dimension $X$. We are interested in the perceived variability on the $X$ dimension. We assume that $X$ follows a normal distribution with mean $\mu_{x}$ and variance $\sigma_{X}: \mu_{X, \text { in }}=\mu_{X, \text { out }}=\mu_{X}$, $\sigma_{X, \text { in }}^{2}=\sigma_{X, \text { out }}^{2}=\sigma_{X}$. Similarly, we assume that $A$ follows a normal distribution with mean $\mu_{A}$ and variance $\sigma_{A}: \mu_{A, \text { in }}=$ $\mu_{A, \text { out }}=\mu_{A}, \sigma_{A, \text { in }}^{2}=\sigma_{A, \text { out }}^{2}=\sigma_{A}^{2}$.

Let $A_{t, g}$ denote attitude of the agent toward group $g$ at the end of period $t$. The probability that the agent samples the in-group is given by:

$$
P_{t+1, \text { in }}=l+(1-l) \frac{e^{s A_{t, \text { in }}}}{e^{s A_{t, \text { in }}}+e^{s A_{t, \text { out }}}},
$$

If the agent samples group $g$ in period $t$, two things happen:

1. The agent updates its attitude toward the group. Its new attitude is a weighted average of its previous attitude and the new observation $a_{t, g}$ :

$$
A_{t, g}=(1-b) A_{t-1, g}+b a_{t, g},
$$

where $b \in[0,1]$ and $a_{t, g} \sim N\left(\mu_{A}, \sigma_{A}\right)$.

2. The agent obtains an observation $x_{t, g}$ of the nonattitudinal dimension, $x_{t, g} \sim N\left(\mu_{X}, \sigma_{X}\right)$.

We assume that perceived heterogeneity is the corrected sample variance, as in Model 1 (eq. 10). The results are very similar to those obtained with the model without attitudes. Suppose first that the means are the same $\left(\mu_{X, \text { in }}=\mu_{X, \text { out }}=0\right.$ and $\left.b=0.5, s=3, l=0.5\right)$. After 15 periods we have: $P\left(V_{15 \text {,in }}^{c}>V_{15, \text { out }}^{c}\right)=.56$. This likelihood was .55 with the baseline model (which is equivalent to $s=0$ and $r=l+.5 l$ ). If the means are different (e.g., $\mu_{i n}=0.2$ and $\mu_{\text {out }}=0$ with $b, s, l$ the same as above) the effect is 


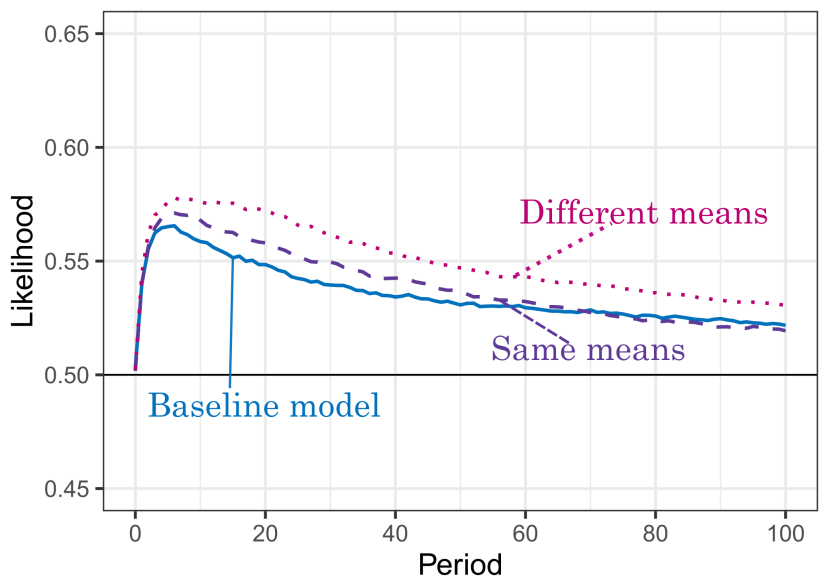

Figure A1. Likelihoods that the estimate of in-group variability is higher than the estimate of out-group variability under hedonic sampling, $P\left(V_{t, \text { in }}^{c}>V_{t, \text { out }}^{c}\right)$, when the estimator of variability is the corrected sample variance (eq. 10). The likelihoods are estimated for three sets of parameters: Baseline model $b=0.5, s=0, \mu_{X, \text { in }}=\mu_{X, \text { out }}=0$ (solid line); Same means $b=0.5, s=3, \mu_{X, \text { in }}=\mu_{X, \text { out }}=0$ (dashed line); Different means $b=0.5, s=3, \mu_{X, \text { in }}=0.2, \mu_{X}$,out $=0$ (dotted line). Based on $10^{5}$ simulations with $l=0.5, \mu_{A}=0, \sigma_{A}^{2}=$ $\sigma_{X, \text { in }}^{2}=\sigma_{X, \text { out }}^{2}=1$.

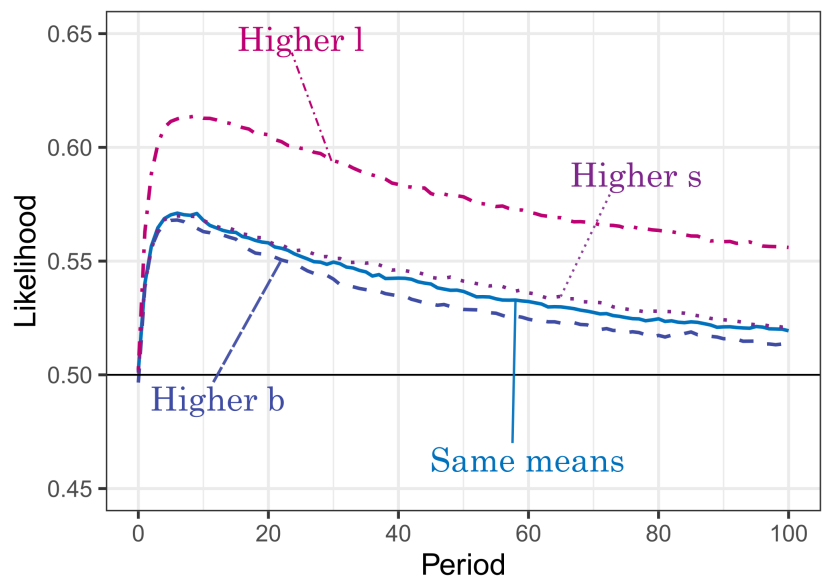

Figure A2. Likelihoods that the estimate of in-group variability is higher than the estimate of out-group variability under hedonic sampling, $P\left(V_{t, \text { in }}^{c}>V_{t, \text { out }}^{c}\right)$, when the estimator of variability is the corrected sample variance (eq. 10). The likelihoods are estimated for four sets of parameters: Same means $b=0.5, s=3, l=0.5$ (solid line); Higher $b$ $b=0.8, s=3, l=0.5$ (dashed line); Higher $s b=0.5, s=$ $8, l=0.5$ (dotted line); Higher $l b=0.5, s=3, l=0.8(\operatorname{dot}$ dashed line). Based on $10^{5}$ simulations with $\mu_{A}=0, \mu_{X}$, in $=$ $\mu_{X, \text { out }}=0, \sigma_{A}^{2}=\sigma_{X, \text { in }}^{2}=\sigma_{X, \text { out }}^{2}=1$.

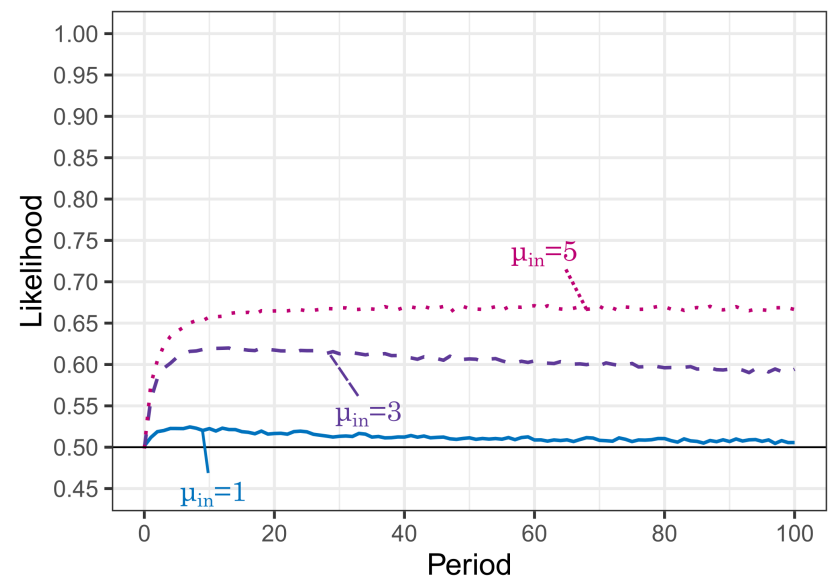

Figure A3. Likelihoods that the estimate of in-group variability is higher than the estimate of out-group variability under similarity-based sampling, $P\left(V_{t, \text { in }}^{c}>V_{t, \text { out }}^{c}\right)$, when the estimator of variability is the corrected sample variance (eq. 10). The likelihoods are estimated for three sets of parameters: $\mu_{X, \text { in }}=1$ (solid line); $\mu_{X, \text { in }}=3$ (dashed line); $\mu_{X, \text { in }}=5$ (dotted line). Based on $10^{5}$ simulations with $\mu_{Y}=0, \sigma_{Y}^{2}=\sigma_{X}^{2}=$ $1, s=3$.

stronger. After 15 periods, we have $P\left(V_{15, \text { in }}^{c}>V_{15, \text { out }}^{c}\right)=.57$ (Figure A1).

The amplitude of the effect depends on $b, s$, and $l$. Higher reliance on the most recent information (higher $b$ ) leads to a weaker in-group heterogeneity effect. At the same time, a higher sensitivity to the current attitude (higher $s$ ) and a larger sampling advantage of the in-group (higher $l$ ) both magnify the effect (Figure A2).

\section{Similarity-Based Sampling of People}

We assume that perceived heterogeneity is the corrected sample variance. Suppose first that the difference in means for feature $X$ in small ( $X$ follows a $N(1,1)$ distribution in the in-group and $N(0,1)$ in the out-group, as described in the body of the paper. After 15 periods we have: $P\left(V_{15, \text { in }}^{c}>V_{15, \text { out }}^{c}\right)=.52$ (with $s=3$ ). When the difference in means is larger ( $X$ follows a $N(5,1)$ distribution in the ingroup and $N(0,1)$ in the out-group), the effect is stronger. After 15 periods we have: $P\left(V_{15, \text { in }}^{c}>V_{15, \text { out }}^{c}\right)=.66$ (with $s=3$; Figure A3).

\section{Groups of Difference Sizes}

We assume that the agent is less likely to sample from the smaller group. In each period, the agent samples from the in-group or the out-group (without replacement). The probability the agent samples a particular member of the in-group is proportional to $r$. Let $k_{t, g}$ denote the number of members 
sampled from group $g$ by the beginning of period $t$ :

$$
k_{t, g}=\sum_{j=-1}^{t-1} I_{j, g}
$$

where $I_{j, g}$ is an indicator variable equal 1 if group $g$ is sampled in period $j$ (and equal to 0 otherwise). The probability the agent samples the in-group in period $t$ is

$$
P_{t+1, \text { in }}=\frac{r\left(N_{\text {in }}-k_{t, \text { in }}\right)}{r\left(N_{\text {in }}-k_{t, \text { in }}\right)+(1-r)\left(N_{\text {out }}-k_{t, \text { out }}\right)} .
$$

This probability decreases with $k_{t, \text { in }}$ and increases with $k_{t, \text { out }}$. That is, for all periods after $t$ such that $k_{t, \text { in }}=N_{\text {in }}$ the probability becomes 0 . At the same time for all periods after $t$ such that $k_{t, \text { out }}=N_{\text {out }}$ the probability becomes 1 . We assume that perceived group variability is given by the corrected sample variance (eq. 10). We consider two cases:
First, suppose the out-group is smaller than the ingroup. We assume $r=.75, N_{\text {in }}=50, N_{\text {out }}=10$. After 15 periods, we have $P\left(V_{15, \text { in }}^{c}>V_{15, \text { out }}^{c}\right)=.62$. In this case, the fact that the out-group is smaller decreases the probability that the agent would sample from it.

Second, suppose the out-group is larger than the ingroup. We assume $N_{\text {in }}=10$ and $N_{\text {out }}=50$. In this case, the model predictions depend on the sampling advantage of the in-group members. For most values of $r$, an in-group homogeneity effect will emerge. For example, with $r=.75$ after 15 periods $P\left(V_{15, \text { in }}^{c}>V_{15, \text { out }}^{c}\right)=.47<.5$. But for very high values of $r$, the sampling advantage compensates for the asymmetry in group sizes. For example, with $r=.9$, we get $P\left(V_{15, \text { in }}^{c}>V_{15, \text { out }}^{c}\right)=.5$.

\section{Appendix B}

Experiments - Additional results 
Table B1

Experiment 1: Proportion of Participants who Indicated the Large Sample School as the More Variable School. $V_{L}$ is the corrected sample variance of the large sample school, $V_{S}$ is the corrected sample variance of the small sample school. $N$ is the number of participants.

\begin{tabular}{c|c|c|c|c}
\hline Question & $\begin{array}{c}\text { All } \\
\text { observations }\end{array}$ & $\begin{array}{c}\text { Conditional on } \\
V_{L}>V_{S}\end{array}$ & $\begin{array}{c}\text { Conditional on } \\
V_{L}<V_{S}\end{array}$ & $\begin{array}{c}\text { Difference } \\
\text { in proportions }\end{array}$ \\
\hline Aggregate & $\begin{array}{c}.58 \\
{[.54, .61]}\end{array}$ & $\begin{array}{c}.63 \\
{[.58, .68]}\end{array}$ & $\begin{array}{c}.50 \\
{[.44, .56]}\end{array}$ & $\begin{array}{c}.13 \\
{[.05, .21]}\end{array}$ \\
\hline Q1 & .55 & .60 & .48 & .12 \\
& {$[.51, .59]$} & {$[.55, .65]$} & {$[.42 .54]$} & {$[.04, .20]$} \\
\hline Q2 & .58 & .62 & .53 & .08 \\
& {$[.54, .62]$} & {$[.57, .67]$} & {$[.47, .59]$} & {$[.01, .17]$} \\
\hline Q3 & .55 & .59 & .49 & .10 \\
& {$[.51, .59]$} & {$[.54, .64]$} & {$[.43, .55]$} & {$[.02, .18]$} \\
\hline$N$ & 650 & 367 & 283 & - \\
Proportion & - & .56 & .44 & - \\
\hline
\end{tabular}

Note: $95 \%$ confidence intervals are in brackets.

Table B2

Results of Experiment 2. $V_{L}$ is the corrected sample variance of the large sample school, $V_{S}$ is the corrected sample variance of the small sample school. $N$ is the number of participants.

\begin{tabular}{c|c|c|c|c|c|c}
\hline & & Aggregate & $\mathrm{Q} 1$ & $\mathrm{Q} 2$ & $\mathrm{Q} 3$ & $N$ \\
\hline Proportion of participants & (Large in-group & .62 & .60 & .66 & .51 & 702 \\
who indicate & sample condition) & {$[.59, .66]$} & {$[.56, .64]$} & {$[.62, .69]$} & {$[.48, .55]$} & - \\
the in-group as & (Small in-group & .39 & .45 & .39 & .41 & 701 \\
more variable & sample condition) & {$[.36, .43]$} & {$[.41, .48]$} & {$[.35, .42]$} & {$[.37, .44]$} & - \\
\hline Proportion of participants & (conditional & .65 & .60 & .66 & .61 & 799 \\
who indicate & on $\left.V_{L}>V_{S}\right)$ & {$[.62, .69]$} & {$[.56, .63]$} & {$[.63, .69]$} & {$[.58, .65]$} & \\
the large-sample school & $($ conditional & .56 & .55 & .60 & .48 & 604 \\
as more variable & on $\left.V_{L}<V_{S}\right)$ & {$[.52, .60]$} & {$[.52, .59]$} & {$[.56, .64]$} & {$[.44, .52]$} & - \\
\hline Difference in proportions & & .09 & 0.05 & 0.06 & 0.13 & - \\
& & {$[.04, .14]$} & {$[-.01, .10]$} & {$[.01, .11]$} & {$[.08, .19]$} & - \\
\hline
\end{tabular}

Note: $95 \%$ confidence intervals are in brackets. 